\title{
Explicitly correlated second-order Møller-Plesset perturbation theory in a Divide- Expand-Consolidate (DEC) context
}

Yang Min Wang', Christof Hättig, Simen Reine, Edward Valeev, Thomas Kjærgaard, and Kasper Kristensen

Citation: The Journal of Chemical Physics 144, 204112 (2016); doi: 10.1063/1.4951696

View online: http://dx.doi.org/10.1063/1.4951696

View Table of Contents: http://aip.scitation.org/toc/jcp/144/20

Published by the American Institute of Physics

\section{Articles you may be interested in}

Perspective: Explicitly correlated electronic structure theory for complex systems

The Journal of Chemical Physics 146, 080901 (2017); 10.1063/1.4976974

Sparse maps-A systematic infrastructure for reduced-scaling electronic structure methods. II. Linear scaling domain based pair natural orbital coupled cluster theory

The Journal of Chemical Physics 144, 024109 (2016); 10.1063/1.4939030

LoFEx - A local framework for calculating excitation energies: Illustrations using RI-CC2 linear response theory

The Journal of Chemical Physics 144, 224106 (2016); 10.1063/1.4953360

The molecular gradient using the divide-expand-consolidate resolution of the identity second-order Møller-

Plesset perturbation theory: The DEC-RI-MP2 gradient

The Journal of Chemical Physics 145, 024106 (2016); 10.1063/1.4956454

Perturbative triples correction for local pair natural orbital based explicitly correlated $\operatorname{CCSD}\left(\mathrm{F} 12^{*}\right)$ using Laplace transformation techniques

The Journal of Chemical Physics 145, 234107 (2016); 10.1063/1.4972001

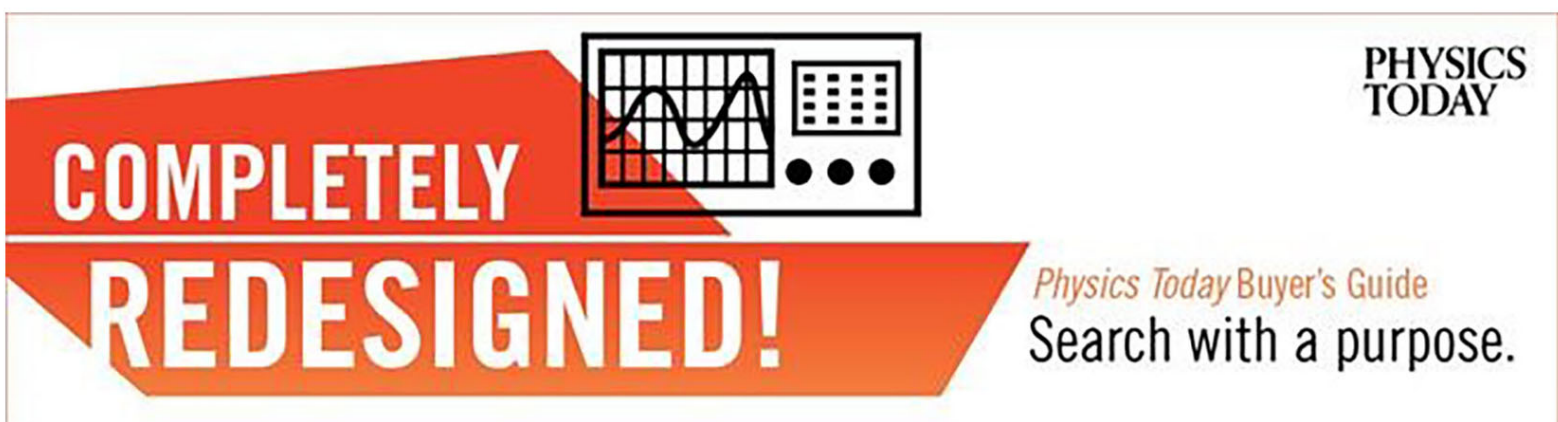




\title{
Explicitly correlated second-order Møller-Plesset perturbation theory in a Divide-Expand-Consolidate (DEC) context
}

\author{
Yang Min Wang, ${ }^{1, a)}$ Christof Hättig, ${ }^{2}$ Simen Reine,${ }^{3}$ Edward Valeev, ${ }^{4}$ Thomas Kjærgaard, ${ }^{1}$ \\ and Kasper Kristensen ${ }^{1}$ \\ ${ }^{1} q L E A P$ Center for Theoretical Chemistry, Department of Chemistry, Aarhus University, \\ Langelandsgade 140, DK-8000 Aarhus C, Denmark \\ ${ }^{2}$ Lehrstuhl für Theoretische Chemie, Ruhr-Universität Bochum, D-44780 Bochum, Germany \\ ${ }^{3}$ Centre for Theoretical and Computational Chemistry, Department of Chemistry, \\ University of Oslo, P.O. Box 1033, N-1315 Blindern, Norway \\ ${ }^{4}$ Department of Chemistry, Virginia Tech, Blacksburg, Virginia 24061, USA
}

(Received 5 February 2016; accepted 8 May 2016; published online 26 May 2016)

\begin{abstract}
We present the DEC-RIMP2-F12 method where we have augmented the Divide Expand-Consolidate resolution-of-the-identity second-order Møller-Plesset perturbation theory method (DEC-RIMP2) [P. Baudin et al., J. Chem. Phys. 144, 054102 (2016)] with an explicitly correlated (F12) correction. The new method is linear-scaling, massively parallel, and it corrects for the basis set incompleteness error in an efficient manner. In addition, we observe that the F12 contribution decreases the domain error of the DEC-RIMP2 correlation energy by roughly an order of magnitude. An important feature of the DEC scheme is the inherent error control defined by a single parameter, and this feature is also retained for the DEC-RIMP2-F12 method. In this paper we present the working equations for the DEC-RIMP2-F12 method and proof of concept numerical results for a set of test molecules. Published by AIP Publishing. [http://dx.doi.org/10.1063/1.4951696]
\end{abstract}

\section{INTRODUCTION}

It is well known that the accurate determination of molecular energies requires an accurate description of electron correlation effects. The coupled cluster (CC) hierarchy has proven to be the most powerful approach for systematically describing dynamical electron correlation. ${ }^{1,2}$ However, the high-order polynomial scaling of the computational cost with system size prohibits the application of CC methods in their standard formulation to large molecular systems. For example, one of the simplest correlation methods, second-order MøllerPlesset perturbation theory (MP2), scales as $O\left(N^{5}\right)$ where $N$ is a measure of the system size. In addition, the slow convergence of the correlation energy for standard basis sets poses a problem in practical calculations. In this work we present a method which tackles the scaling problem as well as the basis set problem of the MP2 model.

In the past few decades much effort has been directed towards developing linear-scaling correlated methods that can handle ever larger molecules. The scaling problem of correlation methods in their standard formulations is to a large part due to the use of de-localized canonical molecular orbitals (MOs) to describe local correlation effects. Several alternative formulations of MP2 and other correlation models with reduced computational scaling have been devised, including local correlation methods based on local occupied MOs and projected atomic orbitals (PAOs), ${ }^{3-7}$ pair natural orbitals (PNOs) ${ }^{8-11}$ or orbital specific virtual orbitals (OSVs). ${ }^{12-14}$ Other local correlation schemes include the fragment orbital method, ${ }^{15-17}$ the incremental scheme, ${ }^{18-20}$ and the

\footnotetext{
a)Electronic mail: ymwang@chem.au.dk
}

cluster-in-molecule (CIM) method. ${ }^{21-23}$ We have recently developed the Divide-Expand-Consolidate (DEC) method, where local occupied and local virtual orbitals are used to partition the correlated energy calculation of a large molecular system into many small and independent fragment calculations employing subsets of the total orbital space. This leads to a linear-scaling and massively parallel implementation of CC methods. ${ }^{24,25,27-32}$ One unique feature of the DEC method is that the error of the correlation energy compared to a conventional calculation is controlled by a single energybased parameter, which determines the local orbital spaces adaptively in a black box manner.

It is well known that the residual error in the correlation energy falls off as $O\left(l_{\max }^{-3}\right)$, where $l_{\max }$ is the maximum angular momentum of the basis set. ${ }^{33-35}$ The reason for this slow basis set convergence is the inability of the wave function to represent the Coulomb cusp, ${ }^{2}$ because conventional wave functions expressed in terms of Slater determinants do not satisfy Kato's cusp condition. ${ }^{36}$ The Coulomb cusp condition can be fulfilled by including the inter-electronic distance operator into the wave-function ansatz. ${ }^{37-40}$ Methods, which explicitly incorporate the inter-electronic distance operator, are referred to as explicitly correlated methods. The most generally applicable explicitly correlated methods, known as F12 methods, have matured into a powerful tool to correct for the basis set incompleteness error (BSIE) which is challenging the results obtained using basis set extrapolation; ${ }^{41,42}$ for recent reviews, see Refs. 43-45. Important contributions to the F12 technology include the fixed amplitude ansatz, ${ }^{46}$ where the F12 contribution becomes a non-iterative correction to the MP2 energy, ${ }^{47}$ and the numerical approximation of many-electron integrals via the complementary auxiliary basis set (CABS) 


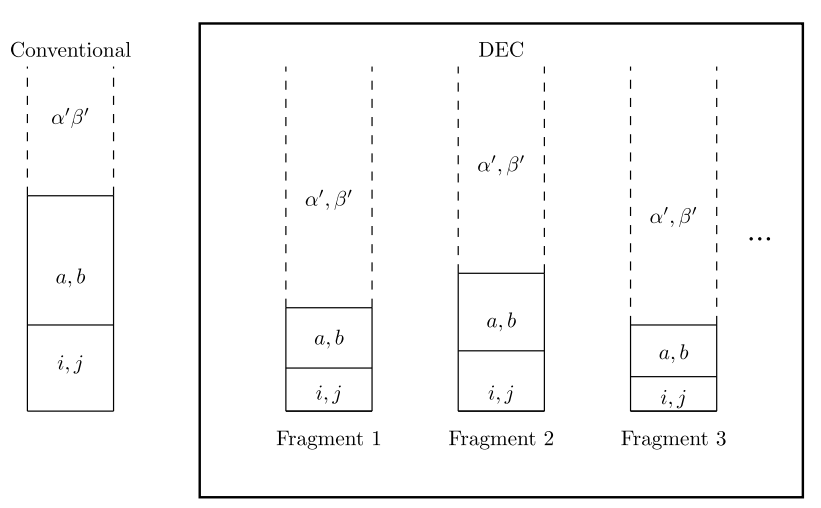

FIG. 1. Comparison of conventional and DEC calculations. In a conventional calculation, the complementary space $\left(\alpha^{\prime} \beta^{\prime}\right)$ spans the space outside the computational basis, which is partitioned into an occupied space $(i, j)$, and a virtual space $(a, b)$. In a DEC calculation there are many fragments, each of which has a different truncated computational basis and thus its own complementary space.

approximation. ${ }^{48,49}$ Furthermore, density fitting ${ }^{50,51}$ is now a widely used tool which reduces the computational costs of evaluating integrals. Several of the reduced scaling correlation methods have been augmented with F12 corrections, ${ }^{52-58}$ and it has been shown that the domain error of the local method can be reduced by the F12 correction. ${ }^{59-62}$

We have recently introduced the Resolution of the Identity (RI) MP2 method into the DEC framework by applying density fitting techniques to all two-electron integrals occurring in the DEC-MP2 method and denoted the resulting method DECRIMP2 ${ }^{63}$ In this work we augment the DEC-RIMP2 method with the MP2-F12 correction often labelled approximation $3 \mathrm{C}^{33,64}$ in combination with the fixed amplitude ansatz, ${ }^{65}$ using density fitting also for the F12 integrals. The resulting DECRIMP2-F12 method retains the linear-scaling and massively parallel features of DEC-RIMP2 and corrects also for the basis set error. The main purpose of the present study is to develop the general theoretical framework necessary for introducing the F12 correction within a DEC context. This is done for the simplest correlated model, RIMP2, in order to set the stage for future developments of more accurate DEC-CC-F12 models, such as the coupled-cluster singles doubles (CCSD) model.

\section{MP2-F12 THEORY}

\section{A. General considerations}

A conventional MP2 calculation is formulated in terms of Hartree-Fock (HF) molecular orbitals (MOs), which are partitioned into a set of occupied $(i, j, k, \ldots)$ and virtual $(a, b, c, \ldots)$ MOs. The MOs are expanded in a finite basis of atomic orbitals (AOs), and we refer to this basis as the computational basis. The philosophy behind the F12 method is to correct for the BSIE by approximately describing the wave function components in the complementary space $\alpha^{\prime}, \beta^{\prime}, \gamma^{\prime}, \ldots$, which is in principle infinite, see Fig. 1 (left). The notation for the different orbital spaces is summarized in Table I.

A DEC calculation consists of many fragment calculations, where each fragment contains a subset of the occupied and virtual orbital spaces for the full system. While in a conventional calculation the orbitals in the complementary space $\alpha^{\prime}, \beta^{\prime}, \gamma^{\prime}, \ldots$ represent the basis set error associated with the use of a finite computational basis, in a DEC calculation the complementary orbitals represent this basis set error as well as the domain error associated with the use of truncated occupied and virtual spaces in the fragment calculations, see Fig. 1 (right). The F12 correction thus becomes even more important in a DEC calculation than in a conventional calculation.

Fig. 1 summarizes the basic philosophy of applying the F12 correction in a DEC context. The specific working equations for the RIMP2-F12 model using the approximation $3 C^{64}$ and the fixed amplitude ansatz ${ }^{65}$ are given in Section II B. These equations provide the necessary framework that allows us to develop the DEC-RIMP2-F12 model in Section III.

\section{B. MP2-F12 theory for a conventional system}

The closed-shell correlation MP2-F12 energy in a real spin-free orbital basis is given by ${ }^{35}$

$$
\begin{aligned}
E_{\mathrm{MP} 2-\mathrm{F} 12} & =E_{\mathrm{MP} 2}+E_{\mathrm{F} 12}, \\
E_{\mathrm{MP} 2} & =t_{a b}^{i j}\left(2 g_{i j}^{a b}-g_{j i}^{a b}\right),
\end{aligned}
$$

where the Einstein summation convention has been used and will be used throughout this paper (unless stated otherwise). $E_{\mathrm{MP} 2}$ is the standard MP2 energy, where $g_{i j}^{a b}$ is the Coulomb integral (using the Dirac notation and MOs $\{\phi\}$ )

$$
\begin{aligned}
g_{i j}^{a b} & =\left\langle\phi_{i} \phi_{j}\left|r_{12}^{-1}\right| \phi_{a} \phi_{b}\right\rangle \\
& =\int d r_{1} d r_{2} \phi_{i}\left(r_{1}\right) \phi_{j}\left(r_{2}\right) r_{12}^{-1} \phi_{a}\left(r_{1}\right) \phi_{b}\left(r_{2}\right),
\end{aligned}
$$

and $t_{a b}^{i j}$ are the standard first-order doubles amplitudes, which can be determined from the amplitude equation

$$
g_{i j}^{a b}+f_{a}^{c} t_{c b}^{i j}+f_{b}^{c} t_{a c}^{i j}-t_{a b}^{k j} f_{k}^{i}-t_{a b}^{i k} f_{k}^{j}=0,
$$

TABLE I. Index conventions for the different orbital spaces.

\begin{tabular}{ll}
\hline \hline$\mu, v$ & AOs in computational basis \\
$\mu^{\prime \prime}, v^{\prime \prime}, \rho^{\prime \prime}, \sigma^{\prime \prime}$ & AOs in finite resolution of identity space (CABSAO+) \\
$p, q, r, s$ & MOs in computational basis \\
$i, j, k, l, m, n$ & Occupied MOs in computational basis \\
$a, b, c, d$ & Virtual MOs in computational basis \\
$a^{\prime}, b^{\prime}, c^{\prime}, d^{\prime}$ & MOs in finite complementary virtual basis (CABSMO) \\
$\alpha, \beta, \gamma, \delta$ & Orbitals in auxiliary basis set for density fitting \\
$\alpha^{\prime}, \beta^{\prime}, \gamma^{\prime}, \delta^{\prime}$ & Orbitals in formally complete complementary basis \\
\hline \hline
\end{tabular}


where $f_{i}^{j}$ and $f_{a}^{b}$ are occupied-occupied and virtual-virtual Fock matrix elements. Using the fixed amplitude ansatz ${ }^{65}$ with the approximation $3 \mathrm{C},{ }^{64}$ the $\mathrm{F} 12$ correlation energy correction $E_{\mathrm{F} 12}$ is partitioned into 4 contributions

$$
\begin{aligned}
& E_{\mathrm{F} 12}=E_{\mathrm{F} 12}^{V}+E_{\mathrm{F} 12}^{C}+E_{\mathrm{F} 12}^{B}+E_{\mathrm{F} 12}^{X}, \\
& E_{\mathrm{F} 12}^{V}=\frac{5}{4} V_{i j}^{i j}-\frac{1}{4} V_{i j}^{j i}, \\
& E_{\mathrm{F} 12}^{C}=\frac{5}{4} C_{i j}^{a b} t_{a b}^{i j}-\frac{1}{4} C_{j i}^{a b} t_{a b}^{i j}+\frac{7}{32} C_{i j}^{a b} \delta t_{a b}^{i j}+\frac{1}{32} C_{j i}^{a b} \delta t_{a b}^{i j}, \\
& E_{F 12}^{X}=-\frac{7}{32}\left(X_{i j}^{i k} f_{k}^{j}+X_{i j}^{k j} f_{k}^{i}\right)-\frac{1}{32}\left(X_{i j}^{k i} f_{k}^{j}+X_{i j}^{j k} f_{k}^{i}\right) \text {, } \\
& E_{\mathrm{F} 12}^{B}=\frac{7}{32} B_{i j}^{i j}+\frac{1}{32} B_{i j}^{j i} .
\end{aligned}
$$

The doubles amplitudes $\delta t_{i j}^{a b}$ are determined by solving the amplitude equation

$$
C_{i j}^{a b}+f_{a}^{c} \delta t_{c b}^{i j}+f_{b}^{c} \delta t_{a c}^{i j}-\delta t_{a b}^{k j} f_{k}^{i}-\delta t_{a b}^{i k} f_{k}^{j}=0,
$$

and the intermediates are given in Ref. 47

$$
\begin{aligned}
V_{i j}^{k l} & =\left\langle\phi_{i} \phi_{j}\left|f_{12} \hat{Q}_{12} r_{12}^{-1}\right| \phi_{k} \phi_{l}\right\rangle, \\
C_{i j}^{p q} & =\left\langle\phi_{i} \phi_{j}\left|f_{12} \hat{Q}_{12}\left(\hat{F}_{1}+\hat{F}_{2}\right)\right| \phi_{p} \phi_{q}\right\rangle, \\
X_{i j}^{k l} & =\left\langle\phi_{i} \phi_{j}\left|f_{12} \hat{Q}_{12} f_{12}\right| \phi_{k} \phi_{l}\right\rangle, \\
B_{i j}^{k l} & =\left\langle\phi_{i} \phi_{j}\left|f_{12} \hat{Q}_{12}\left(\hat{F}_{1}+\hat{F}_{2}\right) f_{12} \hat{Q}_{12}\right| \phi_{k} \phi_{l}\right\rangle,
\end{aligned}
$$

where $\hat{F}_{1}$ and $\hat{F}_{2}$ are Fock operators for electrons with coordinates $r_{1}$ and $r_{2}$, respectively. We have chosen to solve equations for both the standard MP2 amplitudes $t_{a b}^{i j}$ in Eq. (4) and the $\delta t_{a b}^{i j}$ amplitudes in Eq. (10) to enable a separation of the MP2 and F12 contributions which will prove useful for error analyses, but we note that the terms involving $t_{a b}^{i j}$ and $\delta t_{a b}^{i j}$ may be combined such that only one equation needs to be solved. The intermediate matrix element in Eq. (11) can be written as

$$
V_{i j}^{k l}=\left\langle w_{i j}\left|r_{12}^{-1}\right| \phi_{k} \phi_{l}\right\rangle,
$$

where the two-electron F12-basis functions (geminals) are given by

$$
\left|w_{i j}\right\rangle=\hat{Q}_{12} f_{12}\left|\phi_{i} \phi_{j}\right\rangle
$$

$\hat{Q}_{12}$ is a projection operator that ensures strong orthogonality of the geminals to any product of MOs within the computational basis ${ }^{44}$

$$
\hat{Q}_{12}=\left(1-\hat{O}_{1}\right)\left(1-\hat{O}_{2}\right)-\hat{V}_{1} \hat{V}_{2},
$$

where $\hat{O}$ and $\hat{V}$ projects onto the finite occupied and finite virtual spaces, respectively,

$$
\begin{aligned}
& \hat{O}_{1}=\sum_{i}\left|\phi_{i}\left(r_{1}\right)\right\rangle\left\langle\phi_{i}\left(r_{1}\right)\right|, \\
& \hat{V}_{1}=\sum_{a}\left|\phi_{a}\left(r_{1}\right)\right\rangle\left\langle\phi_{a}\left(r_{1}\right)\right| .
\end{aligned}
$$

The inter-electronic distance function $f_{12}$ can be written as ${ }^{65-67}$

$$
f_{12}=-\frac{1}{\gamma} e^{-\gamma r_{12}} \approx \sum_{\nu=1}^{n} c_{\nu} e^{-\gamma_{\nu} r_{12}^{2}}
$$

where $\gamma$ is a basis set dependent parameter which we have chosen in accordance with the recommendations of Ref. 68. The $c_{v}$ and $\gamma_{v}$ parameters can be determined as described in Ref. 67.

The geminals $\left|w_{i j}\right\rangle$ in Eq. (16) represent a twoelectron basis outside the computational basis, since the $f_{12}$ operator working on $\left|\phi_{i} \phi_{j}\right\rangle$ yields two-electron states in the formerly complete basis, while the $\hat{Q}_{12}$ operator projects out components from the computational basis. The introduction of geminals $\left|w_{i j}\right\rangle$ ultimately allows for an efficient description of the Coulomb cusp in the wave function with a relatively small computational basis.

\section{DEC-RIMP2-F12}

\section{A. DEC-MP2}

In a DEC calculation the HF orbitals are localized ${ }^{69-71}$ and assigned ${ }^{32}$ to atomic sites $P, Q, \ldots$. The set of occupied orbitals $\left\{\phi_{i}\right\}$ and virtual orbitals $\left\{\phi_{a}\right\}$ assigned to $P$ is denoted $\underline{P}$ and $\bar{P}$, respectively. The standard MP2 correlation energy expression in Eq. (2) can be rewritten in terms of atomic fragment and pair fragment contributions

$$
E_{\mathrm{MP} 2}=\sum_{P} E_{P}+\sum_{P>Q} E_{P Q},
$$

where the atomic fragment energy $E_{P}$ and pair fragment energy $E_{P Q}$ are given by

$$
\begin{aligned}
E_{P} & =\sum_{i j \in \underline{\underline{P}}} \sum_{a b} t_{a b}^{i j}\left(2 g_{i j}^{a b}-g_{j i}^{a b}\right), \\
E_{P Q} & =\left[\sum_{\substack{i \in \underline{P} \\
j \in \underline{\underline{Q}}}}+\sum_{\substack{i \in \underline{Q} \\
j \in \underline{\underline{P}}}}\right] \sum_{a b} t_{a b}^{i j}\left(2 g_{i j}^{a b}-g_{j i}^{a b}\right) .
\end{aligned}
$$

At this point the energy expressions have not been approximated, and Eqs. (2) and (21) give identical results. Consider now the Coulomb integrals $g_{i j}^{a b}$ for $i j \in \underline{P}$,

$$
g_{i j}^{a b}=\int d \tau \rho_{i a}\left(r_{1}\right) r_{12}^{-1} \rho_{j b}\left(r_{2}\right),
$$

where the charge distributions are defined by

$$
\rho_{i a}\left(r_{1}\right)=\phi_{i}\left(r_{1}\right) \phi_{a}\left(r_{1}\right) .
$$

The integral in Eq. (24) is non-vanishing only if $\rho_{i a}$ and $\rho_{j b}$ are both non-zero. The $\rho_{i a}\left(\rho_{j b}\right)$ charge distribution falls off exponentially with the distance between $a(b)$ and the center $P$. Thus, virtual orbitals with significant influence on $P$ are restricted to a local domain close to center $P$, denoted $[\bar{P}]$, and we may therefore restrict the summations in Eqs. (22) and (23) in the following manner:

$$
\begin{gathered}
E_{P}=\sum_{i j \in \underline{P}} \sum_{a b \in[\bar{P}]} t_{a b}^{i j}\left(2 g_{i j}^{a b}-g_{j i}^{a b}\right), \\
E_{P Q}=\left[\sum_{\substack{i \in \mathcal{P} \\
j \in \underline{Q}}}+\sum_{\substack{i \in \underline{Q} \\
j \in \underline{P}}}\right] \sum_{a b \in[\bar{P}] \cup[\bar{Q}]} t_{a b}^{i j}\left(2 g_{i j}^{a b}-g_{j i}^{a b}\right) .
\end{gathered}
$$

The amplitudes in DEC calculations are determined by solving the MP2 amplitude equation in Eq. (4) in a restricted orbital 
space, e.g., the amplitudes $t_{a b}^{i j}$ in Eq. (26) are determined in a space with $i j \in[\underline{P}]$ and $a b \in[\bar{P}]$, where $[\underline{P}]([\bar{P}])$ is a set of occupied (virtual) orbitals spatially local to atomic site $P$. The $[\underline{P}]$ and $[\bar{P}]$ spaces are determined adaptively in a black box manner to yield atomic fragment energies to a predefined precision, denoted the fragment optimization threshold (FOT). ${ }^{32}$ Pair fragments are then determined using unions of spaces from atomic fragment calculations. We note that in practice Eq. (4) for each fragment is trivially solved in a pseudocanonical basis, and the resulting amplitudes are then transformed to the local basis where the atomic (or pair) fragment energy is evaluated, see Ref. 63 for details.

A localized MO $\phi_{r}^{P}$ assigned to center $P$ can be written as

$$
\phi_{r}^{P}=\sum_{\mu} \chi_{\mu} C_{\mu r}^{P},
$$

where the index $\mu$ runs over all AOs in the computational basis (see Table I). To avoid calculating AO integrals in the full computational basis, we approximate the orbital $\phi_{r}^{P}$ by an orbital $\tilde{\phi}_{r}^{P}$ given by

$$
\tilde{\phi}_{r}^{P}=\sum_{\mu \in\{P\}_{\mathrm{AO}}} \chi_{\mu} \tilde{C}_{\mu r}^{P},
$$

where the atomic fragment extent $\{P\}_{\mathrm{AO}}$ is a restricted set of atomic sites located close to atomic site $P$. The MO coefficients $\tilde{C}_{\mu r}^{P}$ are determined such that $\tilde{\phi}_{r}^{P}$ resembles $\phi_{r}^{P}$ as much as possible in a least square sense. Details regarding the determination of $\{P\}_{\mathrm{AO}}$ and $\tilde{C}_{\mu r}^{P}$ are given in Ref. 32 .

The number of atomic fragment calculations scales linearly with the system size, while the number of pair fragment calculations formally scales quadratically with the system size. However, the pair fragment energies for distant pairs describe dispersion effects, which decay with the inverse pair distance to the sixth power. ${ }^{29}$ This can be used to screen away pairs that are well separated in space without affecting the overall precision. ${ }^{25,28}$ In this way, the total number of fragments and thus the total computational time can be reduced to scale linearly with system size for large systems. We also note that the DEC method is massively parallelizable, since the fragment calculations are independent.

\section{B. DEC-MP2-F12}

In a conventional F12 calculation, the basis functions for the full molecular system is divided into a regular AO space (the computational basis) and a CABSAO space, and the union of these spaces are denoted the CABSAO+ space, ${ }^{49}$ see Fig. 2 (left). The CABSMO basis is then determined as the subset of $\mathrm{CABSAO}+$, which is orthogonal to the computational basis.

As described in Section III A, in a DEC-MP2 calculation for an atomic fragment $P$, we operate with the spaces $[\underline{P}]$ (occupied space), $[\bar{P}]$ (virtual space MOs), and $\{P\}_{\mathrm{AO}}$ (regular AOs), all of which span subspaces of the total computational basis. In a DEC-MP2-F12 calculation, CABSAO and CABSMO spaces are also needed. The CABSAO space for atomic fragment $P$ is created by including all CABSAO orbitals assigned to atomic sites in the $\{P\}_{\text {AO }}$ space. The resulting space is denoted $\{P\}_{\text {CABSAO }}$, while the union of the

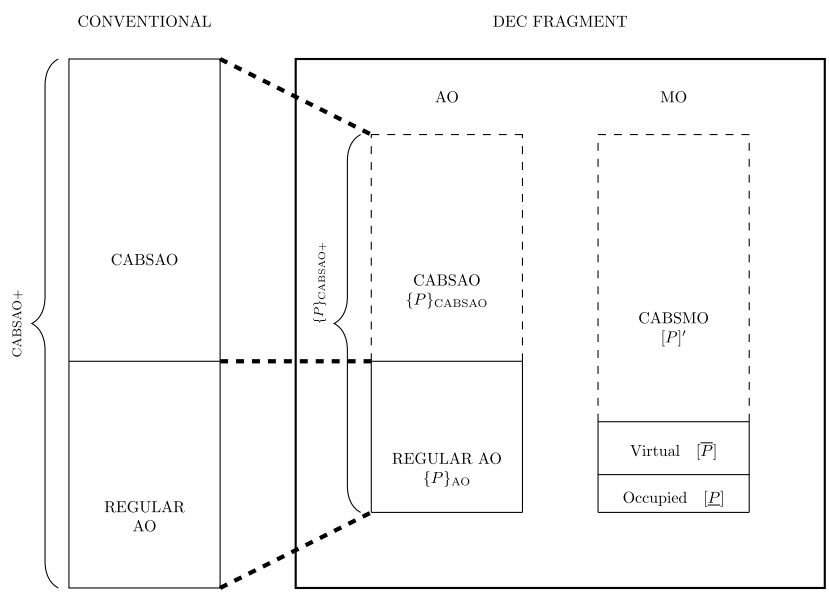

FIG. 2. In a conventional calculation the CABSAO+ space consists of a regular $\mathrm{AO}$ and a CABSAO, while for a DEC fragment the regular $\mathrm{AO}$ and CABSAO are subsets of those in the full calculation. For atomic fragment $\mathrm{P}$, the CABSMO $[P]^{\prime}$ is constructed by orthogonalizing the fragment $\mathrm{CAB}$ $\mathrm{SAO}+,\{P\}_{\mathrm{CABSAO}+}$, against the fragment MO space, $[\underline{P}] \cup[\bar{P}]$.

$\{P\}_{\text {AO }}$ and $\{P\}_{\text {CABSAO }}$ orbitals is denoted the $\{P\}_{\text {CABSAO+ }}$ space. The CABSMO space $[P]^{\prime}$ is then determined as the subset of the $\{P\}_{\text {CABSAO+ }}$ space, which is orthogonal to the union of the occupied and virtual MO spaces for the fragment, $[\underline{P}] \cup[\bar{P}]$, see Fig. 2 (right).

The CABSMO space in a DEC calculation contains some parts of the regular AO basis, since it is defined as the space orthogonal to the MO space $[\underline{P}] \cup[\bar{P}]$, which is generally smaller than $\{P\}_{\mathrm{AO}}$, see Fig. 2 . This is not the case in a conventional calculation, where the regular AO basis spans the same space as the union of the occupied and virtual MO spaces. We also note that the CABSMO for atomic fragment $P$ may contain very small components from occupied orbitals outside $[\underline{P}]$. This effectively implies that the geminals in Eq. (16) may contain small components of occupied orbitals $i \notin[\underline{P}]$, which would not be present in a conventional calculation. However, the results in Section IV indicate that the errors associated with this effect are negligible compared to the precision defined by the FOT. The F12 energy expression in Eq. (5) is significantly more involved than the standard MP2 energy expression in Eq. (2), but we will now use locality arguments to demonstrate that the F12 energy contributions may also be expressed using the DEC partitioning in Eq. (21). To show this we first consider $E_{\mathrm{F} 12}^{V}$ in Eq. (6). By analogy with the standard MP2 case in Eq. (21) we can partition the energy into atomic fragment and pair fragment contributions

$$
E_{\mathrm{F} 12}^{V}=\sum_{P} E_{P}^{V}+\sum_{P<Q} E_{P Q}^{V},
$$

where

$$
\begin{gathered}
E_{P}^{V}=\sum_{i j \in \underline{P}}\left(\frac{5}{4} V_{i j}^{i j}-\frac{1}{4} V_{i j}^{j i}\right), \\
E_{P Q}^{V}=\sum_{\substack{i \in \underline{P} \\
j \in \underline{Q}}}\left(\frac{5}{4} V_{i j}^{i j}-\frac{1}{4} V_{i j}^{j i}\right)+\sum_{\substack{i \in \underline{Q} \\
j \in \underline{P}}}\left(\frac{5}{4} V_{i j}^{i j}-\frac{1}{4} V_{i j}^{j i}\right) .
\end{gathered}
$$


The explicit expressions for the $V$-matrix can be written as ${ }^{44}$

$$
V_{i j}^{i j}=(g r)_{i j}^{i j}-r_{i j}^{p q} g_{p q}^{i j}-r_{i j}^{m a^{\prime}} g_{m a^{\prime}}^{i j}-r_{i j}^{a^{\prime} m} g_{a^{\prime} m}^{i j},
$$

and $E_{\mathrm{F} 12}^{V}$ can thus be partitioned into four contributions, $E^{V 1}, \ldots, E^{V 4}$,

$$
\begin{aligned}
E_{\mathrm{F} 12}^{V} & =E^{V 1}+E^{V 2}+E^{V 3}+E^{V 4}, \\
E^{V 1} & =\frac{5}{4}(g r)_{i j}^{i j}-\frac{1}{4}(g r)_{i j}^{j i}, \\
E^{V 2} & =-\frac{5}{4} r_{i j}^{p q} g_{p q}^{i j}+\frac{1}{4} r_{i j}^{p q} g_{p q}^{j i}, \\
E^{V 3} & =-\frac{5}{4} r_{i j}^{m a^{\prime}} g_{m a^{\prime}}^{i j}+\frac{1}{4} r_{i j}^{m a^{\prime}} g_{m a^{\prime}}^{j i}, \\
E^{V 4} & =-\frac{5}{4} r_{i j}^{a^{\prime} m} g_{a^{\prime} m}^{i j}+\frac{1}{4} r_{i j}^{a^{\prime} m} g_{a^{\prime} m}^{j i} .
\end{aligned}
$$

In addition to the $g_{i j}^{a b}$ integral entering standard MP2 calculations (see Eq. (4)), the $V$-terms also involve integrals of the types $r_{i j}^{p q}$ and $(g r)_{i j}^{p q}$

$$
\begin{aligned}
r_{i j}^{p q} & =\iint \rho_{i p}\left(r_{1}\right) f_{12} \rho_{q j}\left(r_{2}\right) \mathrm{d} r_{1} \mathrm{~d} r_{2}, \\
(g r)_{i j}^{p q} & =\iint \rho_{i p}\left(r_{1}\right) f_{12} r_{12}^{-1} \rho_{q j}\left(r_{2}\right) \mathrm{d} r_{1} \mathrm{~d} r_{2} .
\end{aligned}
$$

Using the same arguments as for the Coulomb integral in Eq. (24), it follows that $p$ and $q$ need to be local to $i$ and $j$, respectively, for the integrals in Eqs. (39) and (40) to be non-zero. This result will be used in the following locality approximations.

Each of the terms in Eqs. (35)-(38) may be partitioned as in Eq. (30). The DEC atomic and pair fragment energies for the $V 1$ term in Eq. (35) involve no locality approximations and are written as

$$
\begin{aligned}
E_{P}^{V 1}= & \frac{5}{4} \sum_{\substack{i \in \underline{P} \\
j \in \underline{P}}}(g r)_{i j}^{i j}-\frac{1}{4} \sum_{\substack{i \in \underline{P} \\
j \in \underline{P}}}(g r)_{i j}^{j i}, \\
E_{P Q}^{V 1}= & \frac{5}{4} \sum_{\substack{i \in \underline{P} \\
j \in \underline{\underline{Q}}}}(g r)_{i j}^{i j}-\frac{1}{4} \sum_{\substack{i \in \in \bar{P} \\
j \in \underline{\underline{Q}}}}(g r)_{i j}^{j i} \\
& +\frac{5}{4} \sum_{\substack{i \in \underline{Q} \\
j \in \underline{\underline{P}}}}(g r)_{i j}^{i j}-\frac{1}{4} \sum_{\substack{i \in \underline{Q} \\
j \in \underline{\underline{P}}}}(g r)_{i j}^{j i} .
\end{aligned}
$$

For $E^{V 2}$ in Eq. (36), the $p$ and $q$ indices may be restricted using locality approximations, and then resulting atomic and pair fragment energies may be written as

$$
\begin{aligned}
& E_{P}^{V 2}=-\frac{5}{4} \sum_{\substack{i \in \underline{P} \\
j \in \underline{P}}} \sum_{\substack{p \in[P] \\
q \in[P]}} g_{i j}^{p q} r_{p q}^{i j}+\frac{1}{4} \sum_{\substack{i \in \underline{P} \\
j \in \underline{P}}} \sum_{\substack{p \in[P] \\
q \in[P]}} g_{i j}^{p q} r_{p q}^{j i}, \\
& E_{P Q}^{V 2}=-\frac{5}{4} \sum_{\substack{i \in \underline{P} \\
j \in \underline{Q} \underline{\underline{Q}}}} \sum_{\substack{p \in[P] \cup[Q] \\
q \in[P] \cup[Q]}} g_{i j}^{p q} r_{p q}^{i j}+\frac{1}{4} \sum_{\substack{i \in \underline{P} \\
j \in \underline{Q}}} \sum_{\substack{p \in[P] \cup[Q] \\
q \in[P] \cup[Q]}} g_{i j}^{p q} r_{p q}^{j i} \\
& -\frac{5}{4} \sum_{\substack{i \in \underline{Q} \\
j \in \underline{\underline{P}}}} \sum_{\substack{p \in[P] \cup[Q] \\
q \in[P] \cup[Q]}} g_{i j}^{p q} r_{p q}^{i j}+\frac{1}{4} \sum_{\substack{i \in \underline{Q} \\
j \in \underline{\underline{P}}}} \sum_{\substack{p \in[P] \cup[Q] \\
q \in[P] \cup[Q]}} g_{i j}^{p q} r_{p q}^{j i},
\end{aligned}
$$

where we have defined $[P]$ as the union of the occupied $[P]$ and virtual space $[\bar{P}],[P]=[\underline{P}] \cup[\bar{P}]$. By applying similar locality arguments to the energy contribution in Eq. (37), the atomic fragment and pair fragment energies can be written as

$$
\begin{aligned}
& E_{P}^{V 3}=-\frac{5}{4} \sum_{\substack{i \in \underline{P} \\
j \in \underline{P} \underline{P}}} \sum_{\substack{a^{\prime} \in[P]^{\prime} \\
m \in[\underline{P}]}} g_{i j}^{m a^{\prime}} r_{m a^{\prime}}^{i j}+\frac{1}{4} \sum_{\substack{i \in \underline{P} \\
j \in \underline{P}}} \sum_{\substack{a^{\prime} \in[P]^{\prime} \\
m \in[\underline{P}]}} g_{i j}^{m a^{\prime}} r_{m a^{j i}}^{j i} \\
& E_{P Q}^{V 3}=-\frac{5}{4} \sum_{\substack{i \in \mathcal{P} \\
j \in \underline{\underline{Q}} \underline{\underline{Q}}}} \sum_{\substack{m \in[\underline{P}] \cup[\underline{Q}] \\
a^{\prime} \in[P \cup \underline{Q}]^{\prime}}} g_{i j}^{m a^{\prime}} r_{m a^{\prime}}^{i j} \\
& +\frac{1}{4} \sum_{\substack{i \in \underline{P} \\
j \in \underline{\underline{Q}}}} \sum_{\substack{m \in[P] \cup[\underline{Q}] \\
a^{\prime} \in[P \cup \bar{Q}]^{\prime}}} g_{i j}^{m a^{\prime}} r_{m a^{\prime}}^{j i} \\
& -\frac{5}{4} \sum_{\substack{i \in \underline{Q} \\
j \in \underline{P} \underline{P}}} \sum_{\substack{m \in[\underline{P}] \cup[Q] \\
a^{\prime} \in[P \cup \bar{Q}]^{\prime}}} g_{i j}^{m a^{\prime}} r_{m a^{\prime}}^{i j} \\
& +\frac{1}{4} \sum_{\substack{i \in \underline{Q} \underline{\underline{Q}} \\
j \in \underline{\underline{P}}}} \sum_{\substack{m \in[P] \cup[\underline{\underline{Q}}] \\
a^{\prime} \in[P \cup \underline{Q}]^{\prime}}} g_{i j}^{m a^{\prime}} r_{m a^{\prime}}^{j i}
\end{aligned}
$$

where $[P]^{\prime}$ denotes the CABSMO space (see Fig. 2) and $[P \cup Q]^{\prime}=[P]^{\prime} \cup[Q]^{\prime}$. The same arguments can be applied to the $V 4$ term, where the atomic and pair fragment energies become

$$
\begin{aligned}
& E_{P}^{V 4}=-\frac{5}{4} \sum_{\substack{i \in P \\
j \in \underline{P}}} \sum_{\substack{a^{\prime} \in[P]^{\prime} \\
m \in[\underline{P}]}} g_{i j}^{a^{\prime} m} r_{a^{\prime} m}^{i j}+\frac{1}{4} \sum_{\substack{i \in \underline{P} \\
j \in \underline{P}}} \sum_{\substack{a^{\prime} \in\left[P P^{\prime} \\
m \in[\underline{P}]\right.}} g_{i j}^{a^{\prime} m} r_{a^{\prime} m}^{j i}, \\
& E_{P Q}^{V 4}=-\frac{5}{4} \sum_{\substack{i \in \underline{P} \\
j \in \underline{\underline{Q}} \underline{\underline{Q}}}} \sum_{\substack{m \in[\underline{P}] \cup[\underline{Q}] \\
a^{\prime} \in[P \cup \underline{Q}]^{\prime}}} g_{i j}^{a^{\prime} m} r_{a^{\prime} m}^{i j} \\
& +\frac{1}{4} \sum_{\substack{i \in P \\
j \in \underline{\underline{Q}} \underline{\underline{Q}}}} \sum_{\substack{m \in[P] \cup[\underline{Q}] \\
a^{\prime} \in[P \cup \bar{Q}]^{\prime}}} g_{i j}^{a^{\prime} m} r_{a^{\prime} m}^{j i} \\
& -\frac{5}{4} \sum_{\substack{i \in \underline{Q} \\
j \in \underline{P}}} \sum_{\substack{m \in[P] \cup[Q] \\
a^{\prime} \in[P \cup Q]^{\prime}}} g_{i j}^{a^{\prime} m} r_{a^{\prime} m}^{i j} \\
& +\frac{1}{4} \sum_{\substack{i \in \underline{Q} \underline{\underline{Q}} \\
j \in \underline{\underline{P}}}} \sum_{\substack{m \in[P] \cup[\underline{\underline{Q}}] \\
a^{\prime} \in[P \cup \bar{Q}]^{\prime}}} g_{i j}^{a^{\prime} m} r_{a^{\prime} m}^{j i} .
\end{aligned}
$$

The remaining terms in Eq. (5) may also be expressed using the DEC partitioning scheme (see Appendix), and the total F12 contribution can therefore be partitioned as in Eq. (21),

$$
E_{\mathrm{F} 12}=\sum_{P} E_{P}^{\mathrm{F} 12}+\sum_{P<Q} E_{P Q}^{\mathrm{F} 12} .
$$

In our implementation the $f_{12}$ function is the exponential function in Eq. (20), which decays faster with the interelectronic distance than the Coulomb operator. ${ }^{35}$ Because of this, the pair energy contributions for F12 will decay faster with pair distance than the standard MP2 pair-energies (inverse sixth power). This can be used to screen away pair fragments with small energy contributions without affecting the precision of the calculation. Analogous to the DEC-MP2 model, the total number of fragments can therefore be reduced so that the DEC-MP2-F12 model scales linearly with system size for large systems. We will return to this in Section IV C.

In summary, it is possible to calculate the F12 contribution in terms of a linear-scaling number of independent fragment 
calculations in the same way as for the RIMP2 contribution, cf. Eqs. (21) and (49). Since the fragment calculations are independent, the DEC-RIMP2-F12 scheme becomes massively parallel. However, the price to pay for a massively parallel and linear-scaling algorithm is a significant repetitive overhead (large pre-factor), because many of the same integrals and amplitudes are required for the different fragment calculations. As a rule of thumb (see Ref. 63), if the conventional calculation can be carried out within a reasonable time for a given molecular system, then the DEC scheme usually requires more computational resources than the conventional calculation. For this reason the purpose of the DEC scheme is not to compete with conventional implementations, but rather to enable calculations of molecular systems that are too large to be treated by a conventional implementation. In this paper we perform an error analysis of the DEC-RIMP2-F12 scheme for systems where a conventional calculation can be carried out to justify the validity of the DEC-RIMP2-F12 approximations, while large-scale calculations will be presented elsewhere.

\section{Density fitting}

We use density fitting ${ }^{50}$ (DF) to reduce the computational cost and memory storage for all integrals that enter in Eqs. (21) and (49). The $g_{i j}^{a b}$ integral entering the standard DEC-RIMP2 calculations is thus determined in the following manner:

$$
\begin{gathered}
g_{p q}^{r s} \approx \sum_{\alpha} C_{p r}^{\alpha} C_{q s}^{\alpha}, \\
C_{p r}^{\alpha}=\sum_{\beta}\left(\phi_{p} \phi_{r}\left|r_{12}^{-1}\right| \phi_{\beta}\right)\left(U^{-1 / 2}\right)_{\beta \alpha}, \\
U_{\beta \alpha}=\left(\phi_{\beta}\left|r_{12}^{-1}\right| \phi_{\alpha}\right),
\end{gathered}
$$

where $\phi_{\alpha}$ and $\phi_{\beta}$ are part of the density fitting basis. The DEC-RIMP2-F12 method also involves the following types of integrals:

$$
\begin{gathered}
\left(r^{2}\right)_{p q}^{r s} \approx \sum_{\alpha} D_{p r}^{\alpha} D_{q s}^{\alpha}, \\
D_{p r}^{\alpha}=\sum_{\beta}\left(\phi_{p} \phi_{r}\left|f_{12}^{2}\right| \phi_{\beta}\right)\left(W^{-1 / 2}\right)_{\beta \alpha}, \\
W_{\beta \alpha}=\left(\phi_{\beta}\left|f_{12}^{2}\right| \phi_{\alpha}\right), \\
r_{p q}^{r s} \approx \sum_{\alpha} F_{p r}^{\alpha} F_{q s}^{\alpha}, \\
F_{p r}^{\alpha}=\sum_{\beta}\left(\phi_{p} \phi_{r}\left|f_{12}\right| \phi_{\beta}\right)\left(Z^{-1 / 2}\right)_{\beta \alpha}, \\
Z_{\beta \alpha}=\left(\phi_{\beta}\left|f_{12}\right| \phi_{\alpha}\right),
\end{gathered}
$$

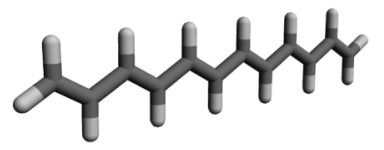

(a)

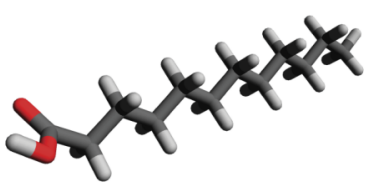

(b)

$$
\begin{gathered}
(g r)_{p q}^{r s} \approx \sum_{\alpha} G_{p r}^{\alpha} G_{q s}^{\alpha}, \\
G_{p r}^{\alpha}=\sum_{\beta}\left(\phi_{p} \phi_{r}\left|r_{12}^{-1} f_{12}\right| \phi_{\beta}\right)\left(Y^{-1 / 2}\right)_{\beta \alpha}, \\
Y_{\beta \alpha}=\left(\phi_{\beta}\left|r_{12}^{-1} f_{12}\right| \phi_{\alpha}\right) .
\end{gathered}
$$

However, one of the integrals is treated differently using robust fitting, ${ }^{51,72}$ since the numerical evaluation of the integral matrix associated with the $\left(\nabla f_{12}\right)^{2}$ operator with $f_{12}$ expanded in Gaussians is not guaranteed to be positive definite

$$
\begin{aligned}
(T)_{p q}^{r s} \approx & \sum_{\alpha} C_{p r}^{\alpha}\left(\phi_{\alpha}\left|\left(\nabla_{1} f_{12}\right)^{2}\right| \phi_{q} \phi_{s}\right) \\
& +\sum_{\alpha}\left(\phi_{p} \phi_{r}\left|\left(\nabla_{1} f_{12}\right)^{2}\right| \phi_{\alpha}\right) C_{q s}^{\alpha} \\
& -\sum_{\alpha} C_{p r}^{\alpha}\left(\phi_{\alpha}\left|\left(\nabla_{1} f_{12}\right)^{2}\right| \phi_{\beta}\right) C_{q s}^{\beta} .
\end{aligned}
$$

In the calculation for the atomic fragment $P$, the density fitting orbitals $\phi_{\alpha}$ and $\phi_{\beta}$ are restricted to atomic sites in the $\{P\}_{\mathrm{AO}}$ space. ${ }^{63}$

\section{RESULTS}

In this section we investigate the performance of the DEC-RIMP2-F12 method. Computational details are given in Section IV A, and the errors of the correlation energy are analysed in Section IV B, while Section IV C is devoted to an analysis of the decay of pair fragment energies.

\section{A. Computational details}

For the proof of concept calculations we have chosen the following set of test molecules (see Fig. 3):

- System A: A conjugated hydrocarbon, dodecahexaene $\left(\mathrm{C}_{12} \mathrm{H}_{14}\right)$.

- System B: A semi-linear, saturated fatty acid, lauric acid $\left(\mathrm{C}_{12} \mathrm{H}_{24} \mathrm{O}_{2}\right)$.

- System C: An $\alpha$-helix structure with three glycine residues $\left(\mathrm{C}_{6} \mathrm{~N}_{3} \mathrm{O}_{4} \mathrm{H}_{11}\right)$.

- System D: A cluster of 12 water molecules $\left(\left(\mathrm{H}_{2} \mathrm{O}\right)_{12}\right)$.

These molecules are not only large enough to see the effects of the DEC approximations (the fragments do not include the whole molecule) but also small enough that the full molecular reference calculations can be carried out. We note that the test set contains a single-bonded carbon chain (B), a conjugated system (A), as well as more three-dimensional systems (C,D)

FIG. 3. Test set of molecules. (a) System A. (b) System B. (c) System C. (d) System D. 
to ensure that systems of different chemical nature are tested. The molecular geometries are given in the supplementary material in Ref. 73.

We have performed two sets of calculations on each system, denoted DZ and TZ. The one referred to as DZ is performed with a cC-pVDZ-F12 basis ${ }^{68,74}$ for the regular AO basis and cc-pVDZ-F12_OPTRI ${ }^{68,74}$ for the CABSAO, with $\gamma=0.9$ in Eq. (20) as recommended in Ref. 68. For the auxiliary basis set, we have constructed a basis denoted aug-cc-pwCVTZ-RI, which is build as the union of three basis sets: cc-pVTZ-RI, ${ }^{68,74}$ aug-cc-pVTZ-RI diffuse, ${ }^{75}$ and cc-pwCVTZ-RI tight. ${ }^{76}$ The TZ calculations use the following combinations: cc-pVTZ-F $12^{68,74}$ for the regular AO basis, cc-pVTZ-F12_OPTRI ${ }^{68,74}$ for the CABSAO with $\gamma=1,{ }^{68}$ and aug-cc-pwCVQZ-RI for the auxiliary basis set. These are standard basis combinations recommended in the literature, which ensure that the remaining basis set incompleteness errors decrease in the sequence orbital basis set error $>$ CABS error $>$ density fitting error (see Ref. 43). The reasoning behind this is that the importance of the size of the basis sets for the computational efficiency decreases in this sequence. All calculations used the frozen core approximation and were carried out using a local version of the quantum chemistry program LSDALTON. ${ }^{26,77}$

\section{B. Correlation energy}

We have performed DEC-RIMP2-F12 calculations and investigated the RIMP2 and F12 correlation energy contributions for different FOTs and compared the results with a conventional calculation. We give the RIMP2, F12, and
RIMP2-F12 energies of the DEC calculations ( $E_{\mathrm{RIMP} 2}, E_{\mathrm{F} 12}$, and $\left.E_{\mathrm{RIMP} 2-\mathrm{F} 12}\right)$ as well as the absolute errors compared to a conventional calculation ( $\Delta E_{\mathrm{RIMP} 2}, \Delta E_{\mathrm{F} 12}$, and $\left.\Delta E_{\mathrm{RIMP} 2-\mathrm{F} 12}\right)$ for different FOTs for the DZ and TZ calculations in Tables II and III, respectively. It is illustrative to plot also the relative RIMP2 and RIMP2-F12 errors, $\Delta E_{\mathrm{RIMP} 2}^{\mathrm{rel}}$ and $\Delta E_{\mathrm{RIMP} 2-\mathrm{r} 12}^{\mathrm{rel}}$, defined as

$$
\begin{array}{r}
\Delta E_{\mathrm{RIMP} 2}^{\mathrm{rel}}=\left|\frac{E_{\mathrm{DEC}-\mathrm{RIMP} 2}(\mathrm{FOT})-E_{\mathrm{RIMP} 2}^{\mathrm{ref}}}{E_{\mathrm{RIMP} 2}^{\mathrm{ref}}}\right|, \\
\Delta E_{\mathrm{RIMP} 2-\mathrm{r} 12}^{\mathrm{rel}}=\left|\frac{E_{\mathrm{DEC}-\mathrm{RIMP} 2-\mathrm{F} 12}(\mathrm{FOT})-E_{\mathrm{RIMP} 2-\mathrm{F} 12}^{\mathrm{ref}}}{E_{\mathrm{RIMP} 2-\mathrm{F} 12}^{\mathrm{ref}}}\right|,
\end{array}
$$

where the "ref" superscript denotes the energy of the conventional reference calculation. The results are given in Figs. 4 and 5.

The general trend in Tables II and III is that both the RIMP2 error $\Delta E_{\mathrm{RIMP} 2}$ and the F12 error $\Delta E_{\mathrm{F} 12}$ are decreasing systematically with the FOT. But more important, the total RIMP2-F12 error $\Delta E_{\mathrm{RIMP} 2-\mathrm{F} 12}$ is about 1-2 orders of magnitude smaller than the RIMP2 error $\Delta E_{\mathrm{RIMP} 2}$ for both the DZ and TZ calculations. This behavior is clearly illustrated in Figs. 4 and 5, although a few deviations from this general trend is observed, for example, for the DZ calculation for system C with FOT $=10^{-2}$ a.u., the RIMP2-F12 error is artificially low due to a fortuitous error cancellation of the $\Delta E_{\mathrm{RIMP} 2}$ and $\Delta E_{\mathrm{F} 12}$ errors. The numerical results in Tables II and III (or Figs. 4 and 5) are in line with the general considerations of Section II A which are summarized in Fig. 1. In a DEC calculation, the F12 contribution corrects both for the basis set incompleteness of the computational

TABLE II. DZ results for the test systems in Fig. 3. The DEC RIMP2, F12, and RIMP2-F12 energies ( $E_{\text {RIMP2, }}$ $E_{\mathrm{F} 12}$, and $\left.E_{\mathrm{RIMP} 2-\mathrm{F} 12}\right)$ as well as the corresponding errors compared to a conventional calculation ( $\Delta E_{\mathrm{RIMP} 2}$,

\begin{tabular}{|c|c|c|c|c|c|c|c|}
\hline System & FOT & $E_{\mathrm{RIMP} 2}$ & $E_{\mathrm{F} 12}$ & $E_{\text {RIMP2-F12 }}$ & $\Delta E_{\mathrm{RIMP} 2}$ & $\Delta E_{\mathrm{F} 12}$ & $\Delta E_{\mathrm{RIMP2}-\mathrm{F} 12}$ \\
\hline \multirow[t]{5}{*}{ A } & $10^{-2}$ & -1.6185 & -0.5021 & -2.1207 & $1.65 \times 10^{-1}$ & $-1.73 \times 10^{-1}$ & $-7.67 \times 10^{-3}$ \\
\hline & $10^{-3}$ & -1.7658 & -0.3508 & -2.1167 & $1.80 \times 10^{-2}$ & $-2.16 \times 10^{-2}$ & $-3.65 \times 10^{-3}$ \\
\hline & $10^{-4}$ & -1.7822 & -0.3307 & -2.1129 & $1.60 \times 10^{-3}$ & $-1.47 \times 10^{-3}$ & $1.32 \times 10^{-4}$ \\
\hline & $10^{-5}$ & -1.7836 & -0.3293 & -2.1130 & $1.57 \times 10^{-4}$ & $-1.26 \times 10^{-4}$ & $3.09 \times 10^{-5}$ \\
\hline & Ref & -1.7838 & -0.3292 & -2.1130 & & & \\
\hline \multirow[t]{5}{*}{ B } & $10^{-2}$ & -2.1751 & -0.6361 & -2.8112 & $1.82 \times 10^{-1}$ & $-1.73 \times 10^{-1}$ & $8.95 \times 10^{-3}$ \\
\hline & $10^{-3}$ & -2.3386 & -0.4800 & -2.8187 & $1.83 \times 10^{-2}$ & $-1.68 \times 10^{-2}$ & $1.48 \times 10^{-3}$ \\
\hline & $10^{-4}$ & -2.3548 & -0.4650 & -2.8199 & $2.13 \times 10^{-3}$ & $-1.81 \times 10^{-3}$ & $3.12 \times 10^{-4}$ \\
\hline & $10^{-5}$ & -2.3568 & -0.4634 & -2.8202 & $2.02 \times 10^{-4}$ & $-1.84 \times 10^{-4}$ & $1.80 \times 10^{-5}$ \\
\hline & Ref & -2.3570 & -0.4632 & -2.8202 & & & \\
\hline \multirow[t]{5}{*}{$\mathrm{C}$} & $10^{-2}$ & -2.2238 & -0.6135 & -2.8373 & $1.28 \times 10^{-1}$ & $-1.26 \times 10^{-1}$ & $2.29 \times 10^{-3}$ \\
\hline & $10^{-3}$ & -2.3326 & -0.5107 & -2.8433 & $1.91 \times 10^{-2}$ & $-2.28 \times 10^{-2}$ & $-3.65 \times 10^{-3}$ \\
\hline & $10^{-4}$ & -2.3501 & -0.4894 & -2.8396 & $1.60 \times 10^{-3}$ & $-1.54 \times 10^{-3}$ & $6.68 \times 10^{-5}$ \\
\hline & $10^{-5}$ & -2.3516 & -0.4880 & -2.8396 & $1.61 \times 10^{-4}$ & $-1.28 \times 10^{-4}$ & $3.27 \times 10^{-5}$ \\
\hline & Ref & -2.3517 & -0.4879 & -2.8396 & & & \\
\hline \multirow[t]{5}{*}{ D } & $10^{-2}$ & -2.9040 & -0.7328 & -3.6368 & $5.84 \times 10^{-2}$ & $-6.28 \times 10^{-2}$ & $-4.46 \times 10^{-3}$ \\
\hline & $10^{-3}$ & -2.9491 & -0.6799 & -3.6290 & $1.32 \times 10^{-2}$ & $-9.89 \times 10^{-3}$ & $3.36 \times 10^{-3}$ \\
\hline & $10^{-4}$ & -2.9608 & -0.6712 & -3.6319 & $1.54 \times 10^{-3}$ & $-1.14 \times 10^{-3}$ & $3.97 \times 10^{-4}$ \\
\hline & $10^{-5}$ & -2.9622 & -0.6701 & -3.6323 & $1.26 \times 10^{-4}$ & $-1.02 \times 10^{-4}$ & $2.43 \times 10^{-5}$ \\
\hline & Ref & -2.9623 & -0.6700 & -3.6323 & & & \\
\hline
\end{tabular}
$\Delta E_{\mathrm{F} 12}$, and $\left.\Delta E_{\mathrm{RIMP2}-\mathrm{F} 12}\right)$ are presented. All energies are given in a.u. 
TABLE III. TZ results for the test systems in Fig. 3. The DEC RIMP2, F12, and RIMP2-F12 energies ( $E_{\text {RIMP2, }}$ $E_{\mathrm{F} 12}$, and $\left.E_{\mathrm{RIMP} 2 \text {-F12) }}\right)$ as well as the corresponding errors compared to a conventional calculation ( $\Delta E_{\mathrm{RIMP} 2}$,

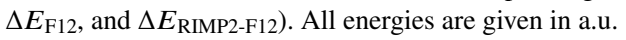

\begin{tabular}{|c|c|c|c|c|c|c|c|}
\hline System & FOT & $E_{\mathrm{RIMP} 2}$ & $E_{\mathrm{F} 12}$ & $E_{\text {RIMP2-F12 }}$ & $\Delta E_{\mathrm{RIMP} 2}$ & $\Delta E_{\mathrm{F} 12}$ & $\Delta E_{\mathrm{RIMP2}-\mathrm{F} 12}$ \\
\hline \multirow[t]{5}{*}{ A } & $10^{-2}$ & -1.8489 & -0.2746 & -2.1236 & $1.28 \times 10^{-1}$ & $-1.26 \times 10^{-1}$ & $2.02 \times 10^{-3}$ \\
\hline & $10^{-3}$ & -1.9597 & -0.1676 & -2.1273 & $1.69 \times 10^{-2}$ & $-1.87 \times 10^{-2}$ & $-1.76 \times 10^{-3}$ \\
\hline & $10^{-4}$ & -1.9752 & -0.1501 & -2.1254 & $1.40 \times 10^{-3}$ & $-1.21 \times 10^{-3}$ & $1.93 \times 10^{-4}$ \\
\hline & $10^{-5}$ & -1.9764 & -0.1491 & -2.1256 & $1.49 \times 10^{-4}$ & $-1.26 \times 10^{-4}$ & $2.26 \times 10^{-5}$ \\
\hline & Ref & -1.9766 & -0.1489 & -2.1256 & & & \\
\hline \multirow[t]{5}{*}{ B } & $10^{-2}$ & -2.4548 & -0.3661 & -2.8208 & $1.71 \times 10^{-1}$ & $-1.54 \times 10^{-1}$ & $1.75 \times 10^{-2}$ \\
\hline & $10^{-3}$ & -2.6051 & -0.2299 & -2.8350 & $2.10 \times 10^{-2}$ & $-1.76 \times 10^{-2}$ & $3.38 \times 10^{-3}$ \\
\hline & $10^{-4}$ & -2.6241 & -0.2137 & -2.8379 & $1.97 \times 10^{-3}$ & $-1.46 \times 10^{-3}$ & $5.17 \times 10^{-4}$ \\
\hline & $10^{-5}$ & -2.6259 & -0.2124 & -2.8383 & $1.88 \times 10^{-4}$ & $-1.47 \times 10^{-4}$ & $4.14 \times 10^{-5}$ \\
\hline & Ref & -2.6261 & -0.2123 & -2.8384 & & & \\
\hline \multirow[t]{5}{*}{$\mathrm{C}$} & $10^{-2}$ & -2.4974 & -0.3509 & -2.8482 & $1.41 \times 10^{-1}$ & $-1.30 \times 10^{-1}$ & $1.11 \times 10^{-2}$ \\
\hline & $10^{-3}$ & -2.6196 & -0.2426 & -2.8622 & $1.90 \times 10^{-2}$ & $-2.18 \times 10^{-2}$ & $-2.86 \times 10^{-3}$ \\
\hline & $10^{-4}$ & -2.6368 & -0.2222 & -2.8591 & $1.71 \times 10^{-3}$ & $-1.43 \times 10^{-3}$ & $2.83 \times 10^{-4}$ \\
\hline & $10^{-5}$ & -2.6384 & -0.2209 & -2.8593 & $1.57 \times 10^{-4}$ & $-1.21 \times 10^{-4}$ & $3.54 \times 10^{-5}$ \\
\hline & Ref & -2.6385 & -0.2208 & -2.8593 & & & \\
\hline \multirow[t]{5}{*}{ D } & $10^{-2}$ & -3.2845 & -0.3721 & -3.6566 & $6.76 \times 10^{-2}$ & $-5.96 \times 10^{-2}$ & $7.98 \times 10^{-3}$ \\
\hline & $10^{-3}$ & -3.3374 & -0.3230 & -3.6605 & $1.47 \times 10^{-2}$ & $-1.05 \times 10^{-2}$ & $4.12 \times 10^{-3}$ \\
\hline & $10^{-4}$ & -3.3508 & -0.3134 & -3.6642 & $1.32 \times 10^{-3}$ & $-9.03 \times 10^{-4}$ & $4.13 \times 10^{-4}$ \\
\hline & $10^{-5}$ & -3.3519 & -0.3126 & -3.6646 & $1.40 \times 10^{-4}$ & $-1.02 \times 10^{-4}$ & $3.78 \times 10^{-5}$ \\
\hline & Ref & -3.3521 & -0.3125 & -3.6646 & & & \\
\hline
\end{tabular}
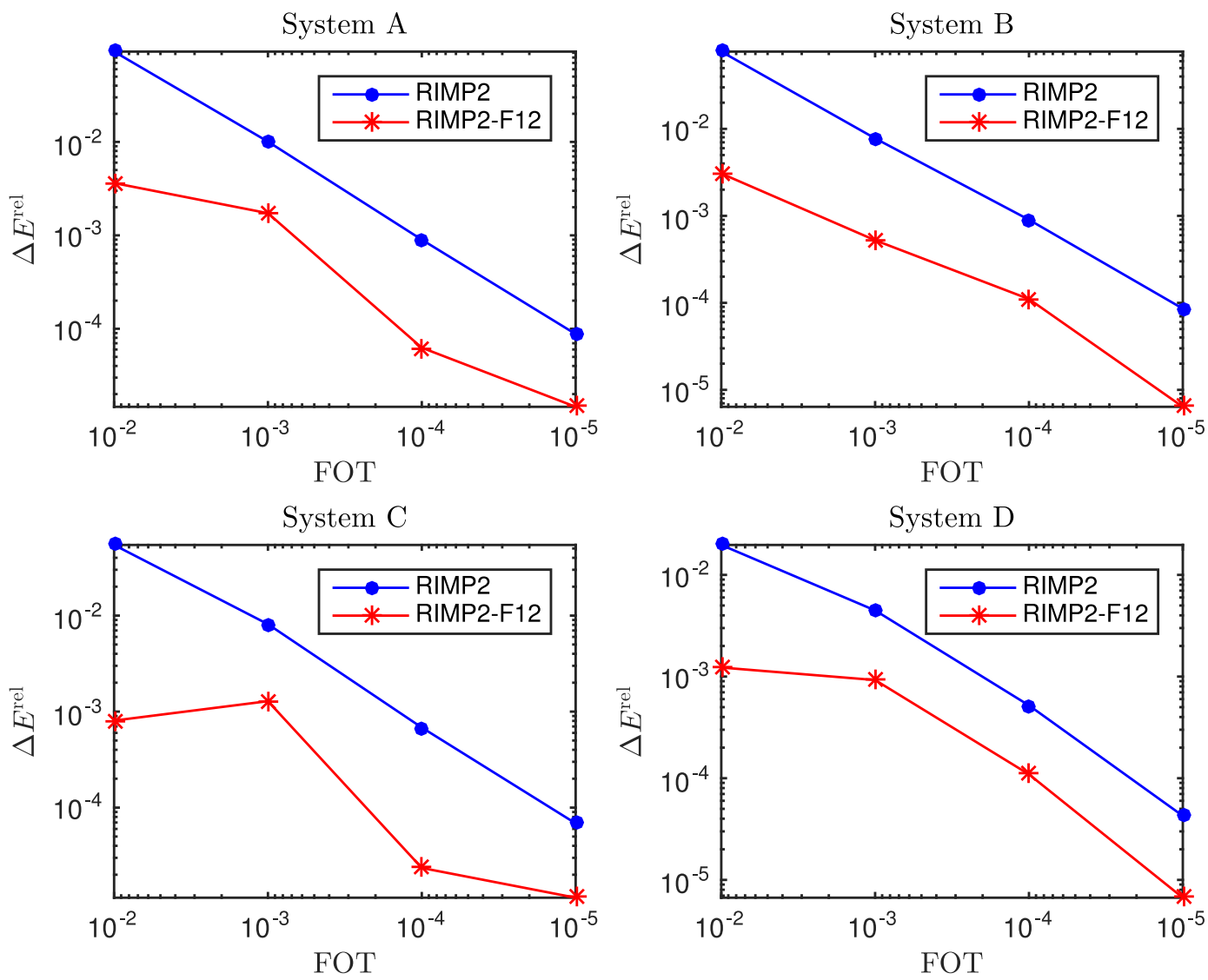

FIG. 4. Relative error plots for the four systems in Fig. 3 for the DZ calculations, see Eqs. (55) and (56). 

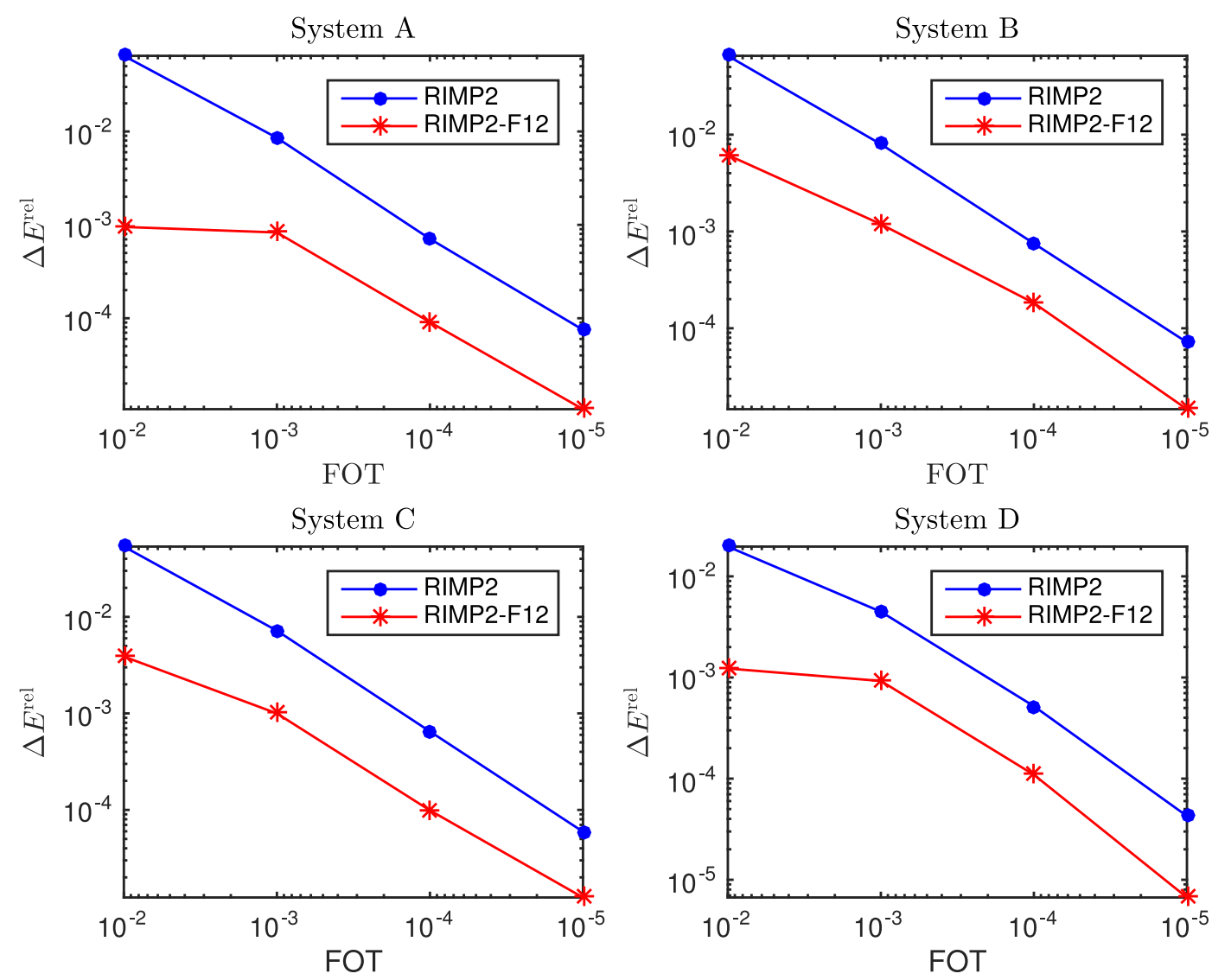

FIG. 5. Relative error plots for the four systems in Fig. 3 for the TZ calculations, see Eqs. (55) and (56).

basis and for the domain error introduced by truncating the local MO spaces of the individual fragment calculations, and the DEC-RIMP2-F12 errors are therefore much smaller than the DEC-RIMP2 errors. This is illustrated by the fact that $\left|E_{\mathrm{F} 12}\right|$ in Tables II and III increases with increasing FOT in order to correct for the larger domain error associated with the RIMP2 contribution. It is quite remarkable that the F12 correction lowers the relative error by about an order of magnitude. This implies that, when the F12 correction is applied in a DEC-RIMP2 calculation, a good accuracy can be obtained using a relatively sloppy FOT value.

We note that Werner and coworkers also found that the F12 correction considerably reduces the error due to truncated PAO domains in the context of their local MP2 (LMP2) method. $^{59,61,62}$ For PNO-based MP2-F12 and CCSD[F12] Schmitz et al. observed a considerably faster decay of the truncation error with the number of PNOs than in the non-F12 methods, but this effect was not present in the PNO implementations for the perturbative correction $\operatorname{CCSD}(2)_{\overline{\mathrm{F} 12}} .{ }^{52}$ In the future publication we will investigate how the $\operatorname{CCSD}(2)_{\overline{\mathrm{F} 12}}$ correction performs in combination with the DEC scheme. Finally, we also note that Pavošević et al. observed that the F12 correction did not reduce the PNO truncation error in their PNO-based MP2-F12 and CCSD-F12 methods, ${ }^{55}$ where the PNOs are not expanded in terms of domains of PAOs for each pair of occupied orbitals, in contrast to PNO-LMP2-F12 scheme of Werner and coworkers. ${ }^{61,62}$

\section{Pair fragment analysis}

In Section IV C 1 we investigate the behaviour of DECRIMP2-F12 pair fragment energies with respect to the pair distance, and in Section IV C 2 we analyze how the F12 correction is distributed among atomic and pair fragments. For this study we have chosen to use palmitic acid $\left(\mathrm{C}_{16} \mathrm{H}_{34} \mathrm{O}_{2}\right.$, the molecular geometry is given in the supplementary material in Ref. 73) using the DZ basis set and $\gamma=0.9$ as described in Section IV A. We consider the special case where all fragments use the full virtual space, but the general conclusions are valid for any choices of FOT.

\section{DEC-RIMP2-F12 pair fragment energies}

The DEC-RIMP2-F12 pair energies $E_{P Q}+E_{P Q}^{\mathrm{F} 12}$ are plotted in Fig. 6, and the immediate observation is that they decay rapidly with pair distance $R_{P Q}$. For large distances the pair energies represent dispersion effects, which decay as $R_{P Q}^{-6}$, as previously shown for MP2 pair energies. ${ }^{28,29}$ In principle, the number of pair fragments scales quadratically with the system size. However, the rapid decay of pair energies in Fig. 6 implies that we can avoid performing calculations for distant pairs without affecting the precision of the final DEC-RIMP2-F12 energy, for example, using a simple distance cutoff. If distant pairs are neglected, the DEC-RIMP2-F12 algorithm becomes linear-scaling. A more sophisticated strategy for screening away negligible pair 


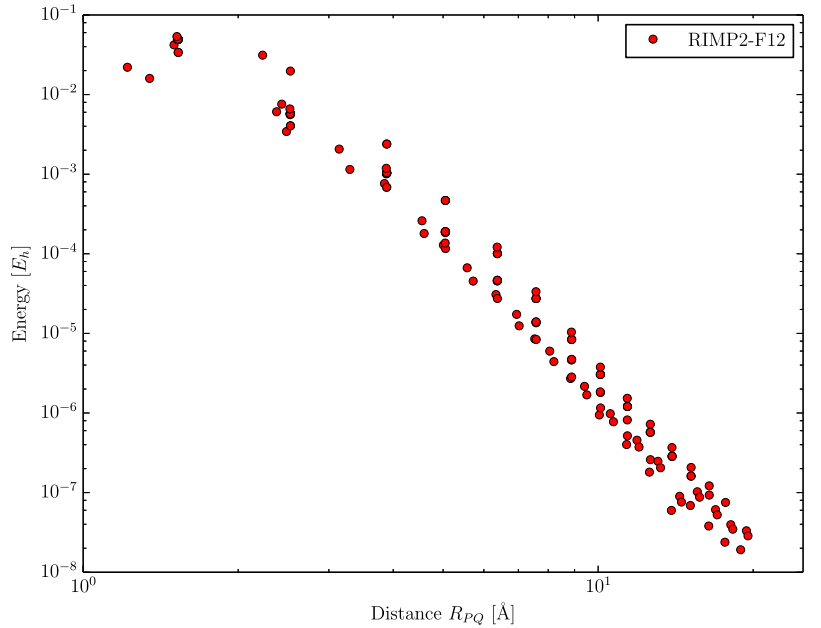

FIG. 6. A loglog plot of the RIMP2-F12 pair fragment energies $E_{P Q}+E_{P Q}^{\mathrm{F} 12}$ as a function of pair distances $R_{P Q}$. The calculation has been carried out on palmitic acid with a DZ basis and $\gamma=0.9$ as described in Section IV A.

fragment contributions will be discussed in a forthcoming paper.

\section{Conceptual analysis of F12 pair fragment energies}

In Fig. 7 we have separated the DEC-RIMP2-F12 pair energies in Fig. 6 into RIMP2 (blue circles) and F12 (red diamonds) contributions. Furthermore, the atomic fragment energies have also been included $\left(R_{P Q}=0\right)$. It is seen that the F12 contribution decays faster than the RIMP2 contribution for larger pair distances. The faster decay of the F12 pair contributions reflects that distant pairs primarily describe dispersion effects which are already well described by the DEC-RIMP2/cc-pVDZ-F12 pair fragment energies.

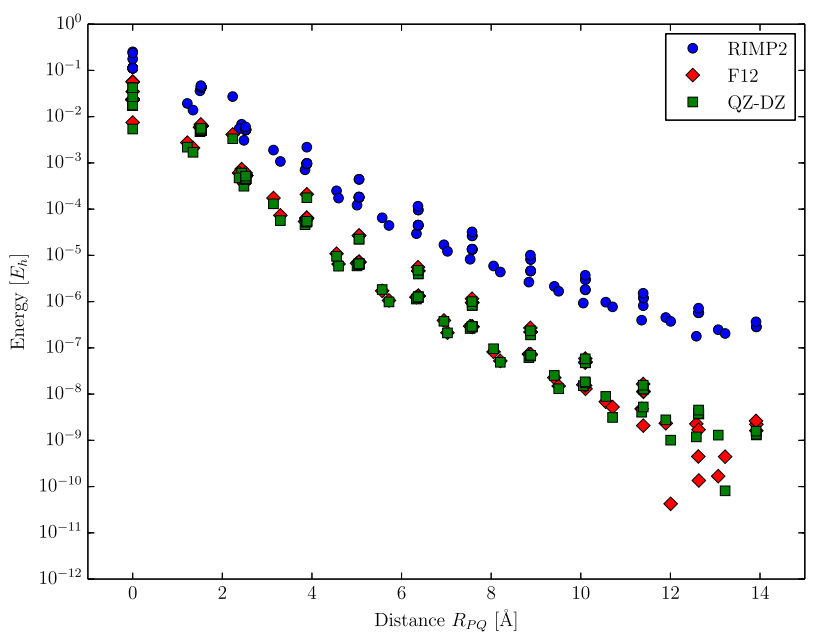

FIG. 7. A semilog plot of the pair fragment energies for RIMP2 (blue circle), F12 (red diamond), and the difference between RIMP2/cc-pVQZ and RIMP2/cc-pVDZ-F12 calculations (QZ-DZ, green square) as a function of pair distances $R_{P Q}$. The calculations were carried out on palmitic acid where we have included the full virtual space for all fragments. The atomic fragment energies are plotted at $R_{P Q}=0$. We note that the data points for two of the atomic sites have been removed, because the QZ-DZ data points were ill-defined due to a difference in the occupied orbital assignment for the cc-pVDZ-F12 and cc-pVQZ basis sets.
Mathematically, this is related to the fact that the $f_{12}$ function decays faster with inter-electronic distance than the Coulomb operator $r_{12}^{-1}$, see the discussion in Section III B.

To get a better understanding of how the F12 correction is distributed among the fragments, it is instructive to calculate a basis set correction to the DEC-RIMP2/cc-pVDZ-F12 energy in an alternative manner. For this purpose we have calculated the absolute energy difference between the DECRIMP2/cc-pVQZ and DEC-RIMP2/cc-pVDZ-F12 fragment energies, which we will refer to as QZ-DZ (green squares in Fig. 7). The F12 and QZ-DZ results in Fig. 7 thus provide two different types of basis set corrections to the DEC-RIMP2/cc-pVDZ-F12 fragment energies. The F12 and QZ-DZ pair energy corrections have a similar distance decay and the atomic fragment energies are also of similar magnitude. The distribution of the F12 energy correction among the atomic and pair fragments is thus similar to the distribution obtained by increasing the cardinal number, and in this sense F12 corrects "uniformly" for the basis set error.

\section{CONCLUSION AND OUTLOOK}

We have introduced the DEC-RIMP2-F12 method by augmenting the DEC-RIMP2 method with an F12 correction. The new method inherits both the linear-scaling and the massively parallel attributes from the parent method. In addition, the DEC-RIMP2-F12 method has error control defined by one threshold (FOT), and both the RIMP2 and F12 errors have been shown to decrease systematically with the FOT. Most importantly, the F12 contribution recovers some of the domain error from the DEC-RIMP2 calculation in addition to the basis set correction, and numerical results have shown that (for a given FOT) the error of the correlation energy is decreased by roughly one order of magnitude when the F12 correction is applied. The present work should be seen as the first step of a development where the F12 correction is introduced into the DEC framework for more advanced correlation methods. For example, we are currently augmenting the DEC-CCSD method with an F12 correction.

\section{ACKNOWLEDGMENTS}

Y.M.W., T.K., and K.K. gratefully acknowledge financial support from the European Research Council under the European Unions Seventh Framework Programme (No. FP/2007-2013)/ERC Grant Agreement No. 291371. C.H. gratefully acknowledges financial support by the Deutsche Forschungsgemeinschaft through Grant No. Ha/2588-7. S.R. gratefully acknowledges the Research Council of Norway (RCN) through CoE Grant No. 179568/V30. The work of E.V. was partially supported by the U.S. National Science Foundation (Award Nos. CHE-0847295 and CHE-1362655) and by Camille and Henry Dreyfus Foundation. This research used resources of the Oak Ridge Leadership Computing Facility at Oak Ridge National Laboratory, which is supported by the Office of Science of the Department of Energy under Contract No. DE-AC05-00OR22725. 


\section{APPENDIX: WORKING EQUATIONS}

In this appendix we present the working equations for the F12 contributions of the DEC-RIMP2-F12 method. The notation for the orbital indices is given in Table I, and the local orbital spaces for an atomic fragment $P$ are illustrated schematically in Fig. 2 (right).

The F12 contribution is written in terms of atomic and pair fragment energy contributions in Eq. (49). The F12 atomic fragment energy $E_{P}^{\mathrm{F} 12}$ and $\mathrm{F} 12$ pair fragment energy $E_{P Q}^{\mathrm{F} 12}$ consist of four contributions (each of which can be further decomposed) which we will denote the $V, C, X$ and $B$ terms, see Eqs. (5)-(9)

$$
\begin{aligned}
& E_{P}^{\mathrm{F} 12}=E_{P}^{V}+E_{P}^{C}+E_{P}^{X}+E_{P}^{B}, \\
& E_{P Q}^{\mathrm{F} 12}=E_{P Q}^{V}+E_{P Q}^{C}+E_{P Q}^{X}+E_{P Q}^{B} .
\end{aligned}
$$

\section{The $\mathrm{V}$ terms}

The expressions for the four $V$-terms are given in the main text in Eqs. (41)-(48).

\section{The $\mathrm{C}$ terms}

The $C$-term in Eq. (7) may be written in terms of three contributions

$$
E^{C_{1}}=\frac{5}{4} g_{i j}^{a^{\prime} b} f_{a^{\prime}}^{a} t_{a b}^{i j}-\frac{1}{4} g_{j i}^{a^{\prime} b} f_{a^{\prime}}^{a} t_{a b}^{i j},
$$

$$
\begin{aligned}
& E^{C_{2}}=\frac{5}{4} g_{i j}^{a a^{\prime}} f_{a^{\prime}}^{b} t_{a b}^{i j}-\frac{1}{4} g_{j i}^{a a^{\prime}} f_{a^{\prime}}^{b} t_{a b}^{i j}, \\
& E^{C_{3}}=\frac{7}{32} C_{i j}^{a b} \delta t_{a b}^{i j}+\frac{1}{32} C_{j i}^{a b} \delta t_{a b}^{i j} .
\end{aligned}
$$

Locality arguments similar to the ones already introduced for the $V$-terms in Section III B can be used to restrict the summations to a local region near the occupied orbitals resulting in the following atomic fragment contributions:

$$
\begin{aligned}
E_{P}^{C 1}= & \frac{5}{4} \sum_{\substack{i \in \underline{P} \\
j \in \underline{P}}} \sum_{\substack{a \in[\bar{P}] \\
b \in[\bar{P}]}} \sum_{a^{\prime} \in[P]^{\prime}} g_{i j}^{a^{\prime} b} f_{a^{\prime}}^{a} t_{a b}^{i j} \\
& -\frac{1}{4} \sum_{\substack{i \in \underline{P} \\
j \in \underline{\underline{P}}}} \sum_{\substack{a \in[\bar{P}] \\
b \in[\bar{P}]}} \sum_{a^{\prime} \in[P]^{\prime}} g_{j i}^{a^{\prime} b} f_{a^{\prime}}^{a} t_{a b}^{i j}, \\
E_{P}^{C 2}= & \frac{5}{4} \sum_{\substack{i \in \underline{P} \\
j \in \underline{P}}} \sum_{\substack{a \in[\bar{P}] \\
b \in[\bar{P}]}} \sum_{a^{\prime} \in[P]^{\prime}} g_{i j}^{a a^{\prime}} f_{a^{\prime}}^{b} t_{a b}^{i j} \\
& -\frac{1}{4} \sum_{\substack{i \in \underline{P} \\
j \in \underline{P}}} \sum_{\substack{a \in[\bar{P}] \\
b \in[\bar{P}]}} \sum_{a^{\prime} \in[P]^{\prime}} g_{j i}^{a a^{\prime}} f_{a^{\prime}}^{b} t_{a b}^{i j}, \\
E_{P}^{C 3}= & \frac{7}{32} \sum_{\substack{i \in \in \\
j \in \underline{P}}} \sum_{\substack{a \in[\bar{P}] \\
b \in[\bar{P}]}} C_{i j}^{a b} \delta t_{a b}^{i j}+\frac{1}{32} \sum_{\substack{i \in \in \\
j \in \underline{P}}} \sum_{\substack{a \in[\bar{P}] \\
b \in[\bar{P}]}} C_{j i}^{a b} \delta t_{a b}^{i j},
\end{aligned}
$$

and the pair fragment energy contributions

$$
\begin{aligned}
& E_{P Q}^{C 1}=\frac{5}{4} \sum_{\substack{i \in \underline{P} \\
j \in \underline{Q}}} \sum_{\substack{a \in[\bar{P}] \cup \bar{Q}] \\
b \in[\bar{P}] \cup \bar{Q}]}} \sum_{a^{\prime} \in[P \cup Q]^{\prime}} g_{i j}^{a^{\prime} b} f_{a^{\prime}}^{a} t_{a b}^{i j}-\frac{1}{4} \sum_{\substack{i \in \underline{P} \\
j \in \underline{\underline{Q}}}} \sum_{\substack{a \in[\bar{P}] \cup \bar{Q}] \\
b \in[\bar{P}] \cup[\bar{Q}]}} \sum_{a^{\prime} \in[P \cup Q]^{\prime}} g_{j i}^{a^{\prime} b} f_{a^{\prime}}^{a} t_{a b}^{i j} \\
& +\frac{5}{4} \sum_{\substack{i \in \underline{Q} \\
j \in \underline{\underline{P}} \underline{\underline{P}}}} \sum_{\substack{a \in[\bar{P}] \cup[\bar{Q}] \\
b \in[\bar{P}] \cup \bar{Q}]}} \sum_{a^{\prime} \in[P \cup Q]^{\prime}} g_{i j}^{a^{\prime} b} f_{a^{\prime}}^{a} t_{a b}^{i j}-\frac{1}{4} \sum_{\substack{i \in \underline{Q} \\
j \in \underline{\underline{Q}}}} \sum_{\substack{a \in[\bar{P}] \cup[\bar{Q}] \\
b \in[\bar{P}] \cup[\bar{Q}]}} \sum_{a^{\prime} \in[P \cup Q]^{\prime}} g_{j i}^{a^{\prime} b} f_{a^{\prime}}^{a} t_{a b}^{i j}, \\
& E_{P Q}^{C 2}=\frac{5}{4} \sum_{\substack{i \in \mathcal{P} \\
j \in \underline{\underline{Q}}}} \sum_{\substack{a \in[\bar{P}] \cup[\bar{Q}] \\
b \in[\bar{P}] \cup \bar{Q}]}} \sum_{a^{\prime} \in[P]^{\prime}} g_{i j}^{a a^{\prime}} f_{a^{\prime}}^{b} t_{a b}^{i j}-\frac{1}{4} \sum_{\substack{i \in \underline{P} \\
j \in \underline{\underline{Q}}}} \sum_{\substack{a \in[\bar{P}] \cup[\bar{Q}] \\
b \in[\bar{P}] \cup \bar{Q}]}} \sum_{a^{\prime} \in[P \cup Q]^{\prime}} g_{j i}^{a a^{\prime}} f_{a^{\prime}}^{b} t_{a b}^{i j} \\
& +\frac{5}{4} \sum_{\substack{i \in \underline{Q} \\
j \in \underline{\underline{P}}}} \sum_{\substack{a \in[\bar{P}] \cup \bar{Q}] \\
b \in[\bar{P}] \cup[\bar{Q}]}} \sum_{a^{\prime} \in[P]^{\prime}} g_{i j}^{a a^{\prime}} f_{a^{\prime}}^{b} t_{a b}^{i j}-\frac{1}{4} \sum_{\substack{i \in \underline{Q} \\
j \in \underline{P}}} \sum_{\substack{a \in[\bar{P}] \cup[\bar{Q}] \\
b \in[\bar{P}] \cup[\bar{Q}]}} \sum_{a^{\prime} \in[P \cup Q]^{\prime}} g_{j i}^{a a^{\prime}} f_{a^{\prime}}^{b} t_{a b}^{i j},
\end{aligned}
$$

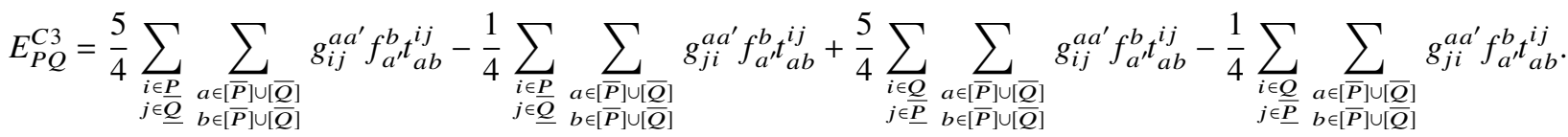

\section{The $\mathbf{X}$ terms}

The $X$-term in Eq. (8) $)^{35}$ consist of four contributions

$$
\begin{aligned}
& E^{X 1}=\frac{7}{32}\left[\left(r^{2}\right)_{i j}^{i k} f_{k}^{j}+\left(r^{2}\right)_{i j}^{k j} f_{k}^{i}\right]+\frac{1}{32}\left[\left(r^{2}\right)_{i j}^{k i} f_{k}^{j}+\left(r^{2}\right)_{i j}^{j k} f_{k}^{i}\right], \\
& E^{X 2}=-\frac{7}{32}\left[r_{i j}^{p q} r_{p q}^{i k} f_{k}^{j}+r_{i j}^{p q} r_{p q}^{k j} f_{k}^{i}\right]-\frac{1}{32}\left[r_{i j}^{p q} r_{p q}^{k i} f_{k}^{j}+r_{i j}^{p q} r_{p q}^{j k} f_{k}^{i}\right],
\end{aligned}
$$




$$
\begin{aligned}
& E^{X 3}=-\frac{7}{32}\left[r_{i j}^{m a^{\prime}} r_{m a^{\prime}}^{i k} f_{k}^{j}+r_{i j}^{m a^{\prime}} r_{m a^{\prime}}^{k j} f_{k}^{i}\right]-\frac{1}{32}\left[r_{i j}^{m a^{\prime}} r_{m a^{\prime}}^{k i} f_{k}^{j}+r_{i j}^{m a^{\prime}} r_{m a^{\prime}}^{j k} f_{k}^{i}\right], \\
& E^{X 4}=-\frac{7}{32}\left[r_{i j}^{a^{\prime} m} r_{a^{\prime} m}^{i k} f_{k}^{j}+r_{i j}^{a^{\prime} m} r_{a^{\prime} m}^{k j} f_{k}^{i}\right]-\frac{1}{32}\left[r_{i j}^{a^{\prime} m} r_{a^{\prime} m}^{k i} f_{k}^{j}+r_{i j}^{a^{\prime} m} r_{a^{\prime} m}^{j k} f_{k}^{i}\right] .
\end{aligned}
$$

By applying locality arguments the atomic fragment contributions become

$$
\begin{aligned}
& E_{P}^{X 1}=\sum_{\substack{i \in \underline{P} \\
j \in \underline{P}}} \sum_{k \in[\underline{P}]}\left(\frac{7}{32}\left[\left(r^{2}\right)_{i j}^{i k} f_{k}^{j}+\left(r^{2}\right)_{i j}^{k j} f_{k}^{i}\right]+\frac{1}{32}\left[\left(r^{2}\right)_{i j}^{k i} f_{k}^{j}+\left(r^{2}\right)_{i j}^{j k} f_{k}^{i}\right]\right), \\
& E_{P}^{X 2}=-\sum_{\substack{i \in P \\
j \in \bar{P} \\
k \in[\underline{P}]}} \sum_{\substack{p \in[P] \\
q \in[P]}}\left(\frac{7}{32}\left[r_{i j}^{p q} r_{p q}^{i k} f_{k}^{j}+r_{i j}^{p q} r_{p q}^{k j} f_{k}^{i}\right]+\frac{1}{32}\left[r_{i j}^{p q} r_{p q}^{k i} f_{k}^{j}+r_{i j}^{p q} r_{p q}^{j k} f_{k}^{i}\right]\right), \\
& E_{P}^{X 3}=-\sum_{\substack{i \in P \\
j \in \bar{P} \\
k \in[\underline{P}]}} \sum_{\substack{m \in[P] \\
a^{\prime} \in[P]^{\prime}}}\left(\frac{7}{32}\left[r_{i j}^{m a^{\prime}} r_{m a^{\prime}}^{i k} f_{k}^{j}+r_{i j}^{m a^{\prime}} r_{m a^{\prime}}^{k j} f_{k}^{i}\right]+\frac{1}{32}\left[r_{i j}^{m a^{\prime}} r_{m a^{\prime}}^{k i} f_{k}^{j}+r_{i j}^{m a^{\prime}} r_{m a^{\prime}}^{j k} f_{k}^{i}\right]\right), \\
& E_{P}^{X 4}=-\sum_{\substack{i \in P \\
j \in P \\
k \in[\underline{P}]}} \sum_{\substack{m \in[P] \\
a^{\prime} \in[P]^{\prime}}}\left(\frac{7}{32}\left[r_{i j}^{a^{\prime} m} r_{a^{\prime} m}^{i k} f_{k}^{j}+r_{i j}^{a^{\prime} m} r_{a^{\prime} m}^{k j} f_{k}^{i}\right]+\frac{1}{32}\left[r_{i j}^{a^{\prime} m} r_{a^{\prime} m}^{k i} f_{k}^{j}+r_{i j}^{a^{\prime} m} r_{a^{\prime} m}^{j k} f_{k}^{i}\right]\right),
\end{aligned}
$$

and the pair fragment contributions are given by

$$
\begin{aligned}
& E_{P Q}^{X 1}=\sum_{\substack{i \in \underline{P} \\
j \in \underline{Q} \\
k \in[\underline{\underline{P}}] \cup[\underline{Q}]}}\left(\frac{7}{32}\left[\left(r^{2}\right)_{i j}^{i k} f_{k}^{j}+\left(r^{2}\right)_{i j}^{k j} f_{k}^{i}\right]+\frac{1}{32}\left[\left(r^{2}\right)_{i j}^{k i} f_{k}^{j}+\left(r^{2}\right)_{i j}^{j k} f_{k}^{i}\right]\right) \\
& +\sum_{\substack{i \in \underline{P} \\
j \in \underline{\underline{Q}} \\
k \in[\underline{[}] \cup \underline{Q}]}}\left(\frac{7}{32}\left[\left(r^{2}\right)_{i j}^{i k} f_{k}^{j}+\left(r^{2}\right)_{i j}^{k j} f_{k}^{i}\right]+\frac{1}{32}\left[\left(r^{2}\right)_{i j}^{k i} f_{k}^{j}+\left(r^{2}\right)_{i j}^{j k} f_{k}^{i}\right]\right), \\
& E_{P Q}^{X 2}=-\sum_{\substack{i \in \boldsymbol{P} \\
j \in \underline{\underline{Q}} \\
k \in[\underline{\underline{Q}}] \cup \underline{Q}]}} \sum_{\substack{p \in[P] \cup[P] \cup[Q] \\
q \in[P]}}\left(\frac{7}{32}\left[r_{i j}^{p q} r_{p q}^{i k} f_{k}^{j}+r_{i j}^{p q} r_{p q}^{k j} f_{k}^{i}\right]-\frac{1}{32}\left[r_{i j}^{p q} r_{p q}^{k i} f_{k}^{j}+r_{i j}^{p q} r_{p q}^{j k} f_{k}^{i}\right]\right) \\
& -\sum_{\substack{i \in \underline{Q} \\
j \in \bar{P} \\
k \in[\underline{P}] \cup \underline{Q}]}} \sum_{\substack{p \in[P] \cup[Q] \\
q \in[P] \cup[Q]}}\left(\frac{7}{32}\left[r_{i j}^{p q} r_{p q}^{i k} f_{k}^{j}+r_{i j}^{p q} r_{p q}^{k j} f_{k}^{i}\right]-\frac{1}{32}\left[r_{i j}^{p q} r_{p q}^{k i} f_{k}^{j}+r_{i j}^{p q} r_{p q}^{j k} f_{k}^{i}\right]\right),
\end{aligned}
$$

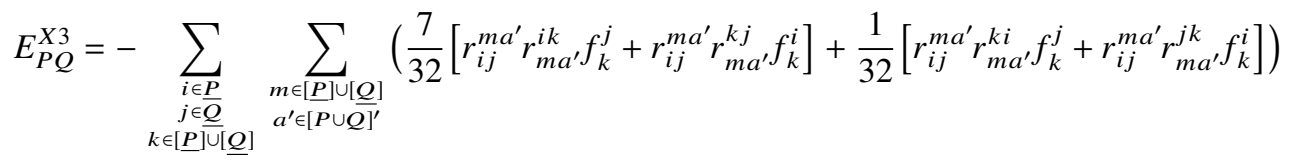

$$
\begin{aligned}
& -\sum_{\substack{i \in \underline{Q} \\
j \in \bar{P} \\
k \in[\underline{P}] \cup \underline{\underline{Q}]}]}} \sum_{\substack{m \in[\underline{P}] \cup[\underline{Q}] \\
a^{\prime} \in[P \cup \underline{Q}]^{\prime}}}\left(\frac{7}{32}\left[r_{i j}^{m a^{\prime}} r_{m a^{\prime}}^{i k} f_{k}^{j}+r_{i j}^{m a^{\prime}} r_{m a^{\prime}}^{k j} f_{k}^{i}\right]+\frac{1}{32}\left[r_{i j}^{m a^{\prime}} r_{m a^{\prime}}^{k i} f_{k}^{j}+r_{i j}^{m a^{\prime}} r_{m a^{\prime}}^{j k} f_{k}^{i}\right]\right),
\end{aligned}
$$

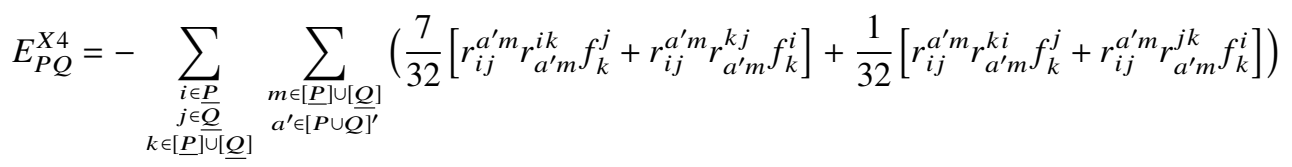

$$
\begin{aligned}
& -\sum_{\substack{i \in \underline{Q} \\
j \in \bar{P} \\
k \in[\underline{P}] \cup \underline{Q}]}} \sum_{\substack{m \in[P] \cup[\underline{Q}] \\
a^{\prime} \in[P \cup \underline{Q}]^{\prime}}}\left(\frac{7}{32}\left[r_{i j}^{a^{\prime} m} r_{a^{\prime} m}^{i k} f_{k}^{j}+r_{i j}^{a^{\prime} m} r_{a^{\prime} m}^{k j} f_{k}^{i}\right]+\frac{1}{32}\left[r_{i j}^{a^{\prime} m} r_{a^{\prime} m}^{k i} f_{k}^{j}+r_{i j}^{a^{\prime} m} r_{a^{\prime} m}^{j k} f_{k}^{i}\right]\right) .
\end{aligned}
$$




\section{The B terms}

The nine $B$-terms originating from Eq. (9) can be found in Ref. 64 as

$$
\begin{aligned}
& E^{B 1}=\frac{7}{32} T_{i j}^{i j}-\frac{1}{32} T_{i j}^{j i}, \\
& E^{B 2}=\frac{7}{32}\left(r^{2}\right)_{i j}^{\mu^{\prime \prime} j}(h J)_{\mu^{\prime \prime}}^{i}+\frac{1}{32}\left(r^{2}\right)_{j i}^{\mu^{\prime \prime j}}(h J)_{\mu^{\prime \prime}}^{i}, \\
& E^{B 3}=\frac{7}{32}\left(r^{2}\right)_{i j}^{i \mu^{\prime \prime}}(h J)_{\mu^{\prime \prime}}^{j}+\frac{1}{32}\left(r^{2}\right)_{i i}^{j \mu^{\prime \prime}}(h J)_{\mu^{\prime \prime}}^{j}, \\
& E^{B 4}=r_{i j}^{\rho^{\prime \prime} \mu^{\prime \prime}} K_{\mu^{\prime \prime}}^{v^{\prime \prime}} r_{\rho^{\prime \prime} v^{\prime \prime}}^{i j}+r_{j i}^{\rho^{\prime \prime} \mu^{\prime \prime}} K_{\mu^{\prime \prime}}^{v^{\prime \prime}} r_{\rho^{\prime \prime} v^{\prime \prime}}^{j i}, \\
& E^{B 5}=-\left(r_{i j}^{p m} f_{\mu^{\prime \prime}}^{v^{\prime \prime}} r_{v^{\prime \prime} m}^{i j}+r_{j i}^{p m} f_{\mu^{\prime \prime \prime}}^{v^{\prime \prime}} r_{v^{\prime \prime} m}^{i}\right), \\
& E^{B 6}=-\left(r_{i j}^{p a} f_{p}^{q} r_{q a}^{i j}+r_{j i}^{p a} f_{p}^{q} r_{q a}^{j i}\right), \\
& E^{B 7}=\left(r_{i j}^{a^{\prime} m} f_{m}^{n} r_{a^{\prime} n}^{i j}+r_{j i}^{a^{\prime} m} f_{m}^{n} r_{a^{\prime} n}^{j i}\right), \\
& E^{B 8}=-2\left(r_{i j}^{a^{\prime} m} f_{m}^{\mu^{\prime \prime}} r_{a^{\prime} \mu^{\prime \prime}}^{i j}+r_{j i}^{a^{\prime} m} f_{m}^{\mu_{m}^{\prime \prime}} r_{a^{\prime} \mu^{\prime \prime}}^{j i}\right), \\
& E^{B 9}=-2\left(r_{i j}^{p a} f_{a}^{a^{\prime}} r_{a^{\prime} a}^{i j}+r_{j i}^{p a} f_{p}^{a^{\prime}} r_{a^{\prime} a}^{j i}\right) .
\end{aligned}
$$

The notation $(h J)_{i}^{j}$ denotes the core Hamiltonian and the Coulomb term of the Fock matrix

$$
(h J)_{i}^{j}=\left\langle\phi_{i}|\hat{h}+\hat{J}| \phi_{j}\right\rangle=\left\langle\phi_{i}|\hat{h}| \phi_{j}\right\rangle+\sum_{m} 2 g_{i m}^{j m},
$$

while $K_{\mu^{\prime \prime}}^{v^{\prime \prime}}$ denotes the exchange term of the Fock matrix

$$
K_{\mu^{\prime \prime}}^{v^{\prime \prime}}=-\sum_{m} g_{\mu^{\prime \prime} m}^{m v^{\prime \prime}}
$$

The intermediate $T_{i j}^{i j}$ entering the first term may be rewritten as

$$
\begin{aligned}
T_{i j}^{i j} & =\left\langle\phi_{i} \phi_{j}\left|\left[\left[f_{12}, \hat{T}_{1}\right], f_{12}\right]\right| \phi_{i} \phi_{j}\right\rangle \\
& =\left\langle\phi_{i} \phi_{j}\left|\left[\left[f_{12}, \hat{T}_{2}\right], f_{12}\right]\right| \phi_{i} \phi_{j}\right\rangle \\
& =\left\langle\phi_{i} \phi_{j}\left|\left(\nabla_{1} f_{12}\right)^{2}\right| \phi_{i} \phi_{j}\right\rangle,
\end{aligned}
$$

where $\hat{T}_{k}$ is the kinetic energy operator for electron $k$. By applying similar locality approximations as for the $V, C$, and
$X$-terms, the $B$-terms may be written as

$$
\begin{aligned}
& E_{P}^{B 1}=\frac{7}{32} \sum_{\substack{i \in \mathcal{P} \\
j \in \underline{P}}} T_{i j}^{i j}+\frac{1}{32} \sum_{\substack{i \in \underline{P} \\
j \in \underline{P}}} T_{i j}^{j i}, \\
& E_{P Q}^{B 1}=\frac{7}{32} \sum_{\substack{i \in \underline{P} \\
j \in \underline{\underline{Q}}}} T_{i j}^{i j}+\frac{1}{32} \sum_{\substack{i \in \underline{P} \\
j \in \underline{\underline{Q}}}} T_{i j}^{j i}+\frac{7}{32} \sum_{\substack{i \in \underline{\underline{Q}} \\
j \in \underline{\underline{P}}}} T_{i j}^{i j}+\frac{1}{32} \sum_{\substack{i \in \underline{\underline{Q}} \\
j \in \underline{\underline{P}}}} T_{i j}^{j i}, \\
& E_{P}^{B 2}=\frac{7}{32} \sum_{\substack{i \in P \\
j \in \underline{P}}} \sum_{\mu^{\prime \prime} \in\{P\}^{\prime \prime}}\left(r^{2}\right)_{i j}^{\mu^{\prime \prime} j}(h J)_{\mu^{\prime \prime}}^{i} \\
& +\frac{1}{32} \sum_{\substack{i \in P \\
j \in P}} \sum_{\mu^{\prime \prime} \in\{P\}^{\prime \prime}}\left(r^{2}\right)_{j i}^{\mu^{\prime \prime} j}(h J)_{\mu^{\prime \prime}}^{i}, \\
& E_{P Q}^{B 2}=\frac{7}{32} \sum_{\substack{i \in P \\
j \in Q}} \sum_{\mu^{\prime \prime} \in\{P \cup Q\}^{\prime \prime}}\left(r^{2}\right)_{i j}^{\mu^{\prime \prime} j}(h J)_{\mu^{\prime \prime}}^{i} \\
& +\frac{1}{32} \sum_{\substack{i \in \underline{P} \\
j \in \underline{Q}}} \sum_{\mu^{\prime \prime} \in\{P \cup Q\}^{\prime \prime}}\left(r^{2}\right)_{j i}^{\mu^{\prime \prime} j}(h J)_{\mu^{\prime \prime}}^{i} \\
& +\frac{7}{32} \sum_{\substack{i \in Q \\
j \in \underline{P}}} \sum_{\mu^{\prime \prime} \in\{P \cup Q\}^{\prime \prime}}\left(r^{2}\right)_{i j}^{\mu^{\prime \prime} j}(h J)_{\mu^{\prime \prime}}^{i} \\
& +\frac{1}{32} \sum_{\substack{i \in \underline{Q} \\
j \in \underline{\underline{P}}}} \sum_{\mu^{\prime \prime} \in\{P \cup Q\}^{\prime \prime}}\left(r^{2}\right)_{j i}^{\mu^{\prime \prime} j}(h J)_{\mu^{\prime \prime}}^{i}
\end{aligned}
$$

$$
\begin{aligned}
E_{P}^{B 3}= & \frac{7}{32} \sum_{\substack{i \in \underline{P} \\
j \in \underline{P}}} \sum_{\mu^{\prime \prime} \in\{P\}^{\prime \prime}}\left(r^{2}\right)_{i j}^{i \mu^{\prime \prime}}(h J)_{\mu^{\prime \prime}}^{j} \\
& +\frac{1}{32} \sum_{\substack{i \in \underline{P} \\
j \in \underline{P}}} \sum_{\mu^{\prime \prime} \in\{P\}^{\prime \prime}}\left(r^{2}\right)_{i i}^{j \mu^{\prime \prime}}(h J)_{\mu^{\prime \prime}}^{j},
\end{aligned}
$$

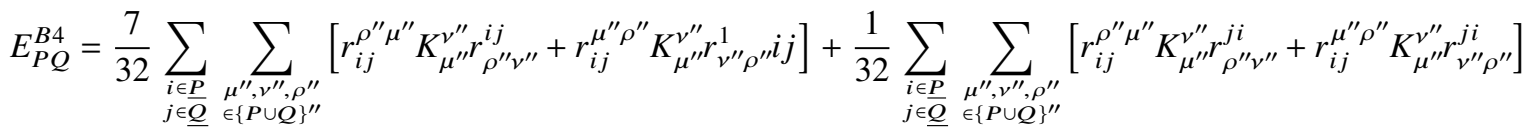

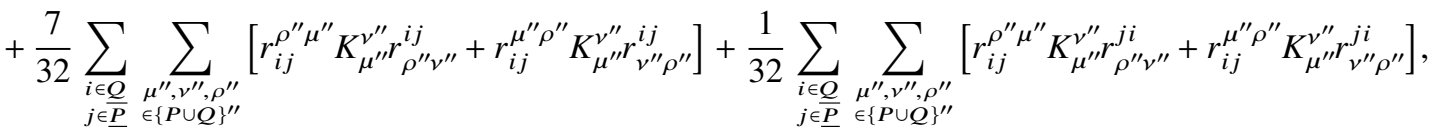




$$
\begin{aligned}
& E_{P}^{B 5}=-\frac{7}{32} \sum_{\substack{i \in \boldsymbol{P} \\
j \in \underline{P}}} \sum_{\substack{\mu^{\prime \prime}, v^{\prime \prime} \in\{\boldsymbol{P}\}^{\prime \prime} \\
m \in[\underline{P}]}}\left[r_{i j}^{\mu^{\prime \prime} m} F_{\mu^{\prime \prime}}^{v^{\prime \prime}} r_{v^{\prime \prime} m}^{i j}+r_{j i}^{\mu^{\prime \prime} m} F_{\mu^{\prime \prime} v_{v^{\prime \prime} m}^{v^{\prime \prime}} r^{j i}}^{j i}-\frac{1}{32} \sum_{\substack{i \in \underline{P} \\
j \in \underline{P}}} \sum_{\substack{\mu^{\prime \prime}, v^{\prime \prime} \in\{P\}^{\prime \prime} \\
m \in[\underline{P}]}}\left[r_{i j}^{\mu^{\prime \prime} m} F_{\mu^{\prime \prime}}^{v^{\prime \prime}} r_{v^{\prime \prime} m}^{i j}+r_{j i}^{\mu^{\prime \prime} m} F_{\mu^{\prime \prime}}^{v^{\prime \prime}} r_{v^{\prime \prime} m}^{j i}\right],\right.
\end{aligned}
$$

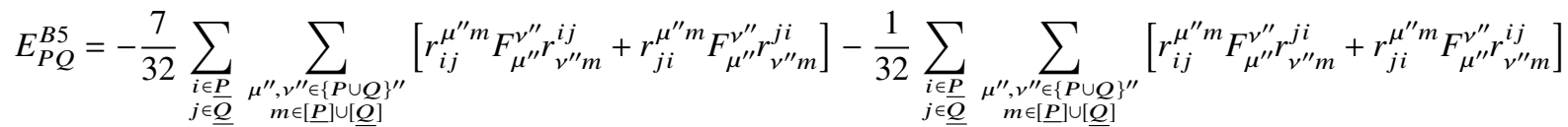

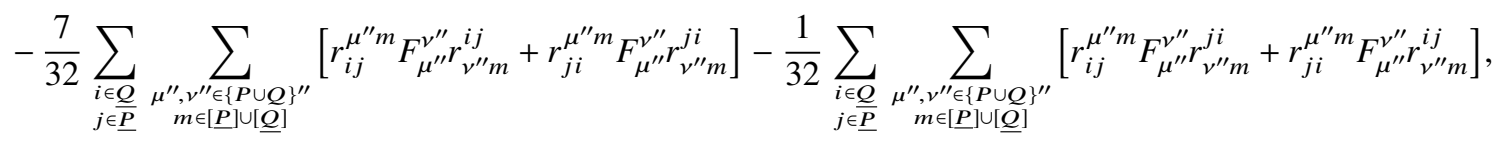

$$
\begin{aligned}
& E_{P}^{B 6}=-\frac{7}{32} \sum_{\substack{i \in \underline{P} \\
j \in \underline{P}}} \sum_{\substack{p, q \in[\bar{P}] \\
a \in[\bar{P}]}}\left[r_{i j}^{p a} F_{p}^{q} r_{q a}^{i j}+r_{j i}^{p a} F_{p}^{q} r_{q a}^{j i}\right]-\frac{1}{32} \sum_{\substack{i \in \underline{P} \\
j \in \underline{P}}} \sum_{\substack{p, q \in[P] \\
a \in[\bar{P}]}}\left[r_{i j}^{p a} F_{p}^{q} r_{q a}^{j i}+r_{j i}^{p a} F_{p}^{q} r_{q a}^{i j}\right],
\end{aligned}
$$

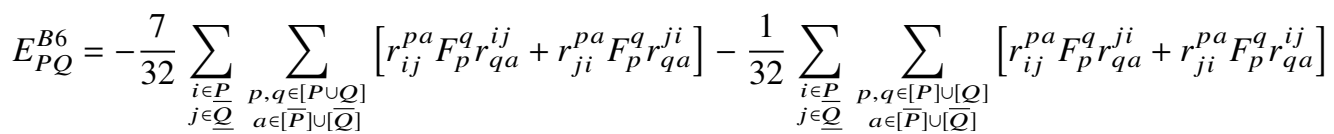

$$
\begin{aligned}
& -\frac{7}{32} \sum_{\substack{i \in \underline{Q} \\
j \in \underline{P}}} \sum_{\substack{p, q \in[P \cup Q] \\
a \in[\bar{P}] \cup[\bar{Q}]}}\left[r_{i j}^{p a} F_{p}^{q} r_{q a}^{i j}+r_{j i}^{p a} F_{p}^{q} r_{q a}^{j i}\right]-\frac{1}{32} \sum_{\substack{i \in \underline{Q} \\
j \in \underline{P}}} \sum_{\substack{p, q \in[P] \cup[Q] \\
a \in[\bar{P}] \cup[\bar{Q}]}}\left[r_{i j}^{p a} F_{p}^{q} r_{q a}^{j i}+r_{j i}^{p a} F_{p}^{q} r_{q a}^{i j}\right], \\
& E_{P}^{B 7}=\frac{7}{32} \sum_{\substack{i \in \underline{P} \\
j \in \underline{P} \underline{\underline{P}}}} \sum_{\substack{m, n \in[\underline{P}] \\
p^{\prime} \in[P]^{\prime}}}\left[r_{i j}^{p^{\prime} m} F_{m}^{n} r_{p^{\prime} n}^{i j}+r_{j i}^{p^{\prime} m} F_{m}^{n} r_{p^{\prime} n}^{j i}\right]+\frac{1}{32} \sum_{\substack{i \in \underline{P} \\
j \in \underline{P}}} \sum_{\substack{m, n \in[\underline{P}] \\
p^{\prime} \in[P]^{\prime}}}\left[r_{i j}^{p^{\prime} m} F_{m}^{n} r_{p^{\prime} n}^{j i}+r_{j i}^{p^{\prime} m} F_{m}^{n} r_{p^{\prime} n}^{i j}\right] \\
& E_{P Q}^{B 7}=\frac{7}{32} \sum_{\substack{i \in \underline{P} \\
j \in \underline{Q}}} \sum_{\substack{m, n \in[\underline{P}] \cup[\underline{Q}] \\
p^{\prime} \in[P \cup Q]^{\prime}}}\left[r_{i j}^{p^{\prime} m} F_{m}^{n} r_{p^{\prime} n}^{i j}+r_{j i}^{p^{\prime} m} F_{m}^{n} r_{p^{\prime} n}^{j i}\right]+\frac{1}{32} \sum_{\substack{i \in \underline{P} \\
j \in \underline{\underline{Q}}}} \sum_{\substack{m, n \in[\underline{P}] \cup[\underline{Q}] \\
p^{\prime} \in[P \cup Q]^{\prime}}}\left[r_{i j}^{p^{\prime} m} F_{m}^{n} r_{p^{\prime} n}^{j i}+r_{j i}^{p^{\prime} m} F_{m}^{n} r_{p^{\prime} n}^{i j}\right] \\
& +\frac{7}{32} \sum_{\substack{i \in \underline{Q} \\
j \in \underline{P}}} \sum_{\substack{m, n \in[\underline{P}] \cup[\underline{Q}] \\
p^{\prime} \in[P \cup Q]^{\prime}}}\left[r_{i j}^{p^{\prime} m} F_{m}^{n} r_{p^{\prime} n}^{i j}+r_{j i}^{p^{\prime} m} F_{m}^{n} r_{p^{\prime} n}^{j i}\right]+\frac{1}{32} \sum_{\substack{i \in \underline{Q} \\
j \in \underline{P}}} \sum_{\substack{m, n \in[\underline{P}] \cup[\underline{Q}] \\
p^{\prime} \in[P \cup Q]^{\prime}}}\left[r_{i j}^{p^{\prime} m} F_{m}^{n} r_{p^{\prime} n}^{j i}+r_{j i}^{p^{\prime} m} F_{m}^{n} r_{p^{\prime} n}^{i j}\right], \\
& E_{P}^{B 8}=-\frac{7}{32} \sum_{\substack{i \in \underline{P} \\
j \in \underline{P}}} \sum_{\substack{p^{\prime} \in[P]^{\prime} \\
\mu^{\prime \prime} \in\{P\}^{\prime \prime} \\
m \in[\underline{P}]}} 2\left[r_{i j}^{p^{\prime} m} F_{m}^{\mu^{\prime \prime}} r_{p^{\prime} \mu^{\prime \prime}}^{i j}+r_{j i}^{p^{\prime} m} F_{m}^{\mu^{\prime \prime}} r_{p^{\prime} \mu^{\prime \prime}}^{j i}\right]-\frac{1}{32} \sum_{\substack{i \in \underline{P} \\
j \in \underline{P}}} \sum_{\substack{p^{\prime} \in[P]^{\prime} \\
\mu^{\prime \prime} \in\{P\}^{\prime \prime} \\
m \in[\underline{P}]}} 2\left[r_{i j}^{p^{\prime} m} F_{m}^{\mu^{\prime \prime}} r_{p^{\prime} \mu^{\prime \prime}}^{j i}+r_{j i}^{p^{\prime} m} F_{m}^{\mu^{\prime \prime}} r_{p^{\prime} \mu^{\prime \prime}}^{i j}\right], \\
& E_{P Q}^{B 8}=-\frac{7}{32} \sum_{\substack{i \in \underline{P} \\
j \in \underline{Q}}} \sum_{\substack{\left.p^{\prime} \in[P \cup Q]^{\prime} \\
\mu^{\prime \prime} \in\{P \cup Q\}^{\prime \prime} \\
m \in[\underline{P}] \cup \underline{Q}\right]}} 2\left[r_{i j}^{p^{\prime} m} F_{m}^{\mu^{\prime \prime}} r_{p^{\prime} \mu^{\prime \prime}}^{i j}+r_{j i}^{p^{\prime} m} F_{m}^{\mu^{\prime \prime}} r_{p^{\prime} \mu^{\prime \prime}}^{j i}\right]-\frac{1}{32} \sum_{\substack{i \in \underline{P} \\
j \in \underline{\underline{Q}}}} \sum_{\substack{p^{\prime} \in[P \cup Q]^{\prime} \\
\mu^{\prime \prime} \in\left\{\cup\{\}^{\prime \prime} \\
m \in[\underline{P}] \cup \underline{Q}\right]}} 2\left[r_{i j}^{p^{\prime} m} F_{m}^{\mu^{\prime \prime}} r_{p^{\prime} \mu^{\prime \prime}}^{j i}+r_{j i}^{p^{\prime} m} F_{m}^{\mu^{\prime \prime}} r_{p^{\prime} \mu^{\prime \prime}}^{i j}\right] \\
& -\frac{7}{32} \sum_{\substack{i \in \underline{Q} \\
j \in \underline{P}}} \sum_{\substack{p^{\prime} \in[P \cup Q]^{\prime} \\
\mu^{\prime \prime} \in\{P \cup Q\}^{\prime \prime} \\
m \in[\underline{P}] \cup[\underline{Q}]}} 2\left[r_{i j}^{p^{\prime} m} F_{m}^{\mu^{\prime \prime}} r_{p^{\prime} \mu^{\prime \prime}}^{i j}+r_{j i}^{p^{\prime} m} F_{m}^{\mu^{\prime \prime}} r_{p^{\prime} \mu^{\prime \prime}}^{j i}\right]-\frac{1}{32} \sum_{\substack{i \in \underline{Q} \\
j \in \underline{P}}} \sum_{\substack{\left.p^{\prime} \in[P \cup Q]^{\prime} \\
\mu^{\prime \prime} \in\{P \cup Q\}^{\prime \prime} \\
m \in[\underline{P}] \cup \underline{Q}\right]}} 2\left[r_{i j}^{p^{\prime} m} F_{m}^{\mu^{\prime \prime}} r_{p^{\prime} \mu^{\prime \prime}}^{j i}+r_{j i}^{p^{\prime} m} F_{m}^{\mu^{\prime \prime}} r_{p^{\prime} \mu^{\prime \prime}}^{i j}\right], \\
& E_{P}^{B 9}=-\frac{7}{32} \sum_{\substack{i \in \bar{P} \\
j \in \underline{P}}} \sum_{\substack{p \in[P] \\
p^{\prime} \in[P]^{\prime} \\
a \in[\bar{P}]}} 2\left[r_{i j}^{p a} F_{p^{\prime}}^{p} r_{p^{\prime} a}^{i j}+r_{j i}^{p a} F_{p^{\prime}}^{p} r_{p^{\prime} a}^{j i}\right]-\frac{1}{32} \sum_{\substack{i \in \underline{P} \\
j \in \underline{P}}} \sum_{\substack{p \in[P] \\
p^{\prime} \in[P]^{\prime} \\
a \in[\bar{P}]}} 2\left[r_{i j}^{p a} F_{p}^{p^{\prime}} r_{p^{\prime} a}^{j i}+r_{j i}^{p a} F_{p}^{p^{\prime}} r_{p^{\prime} a}^{i j}\right],
\end{aligned}
$$

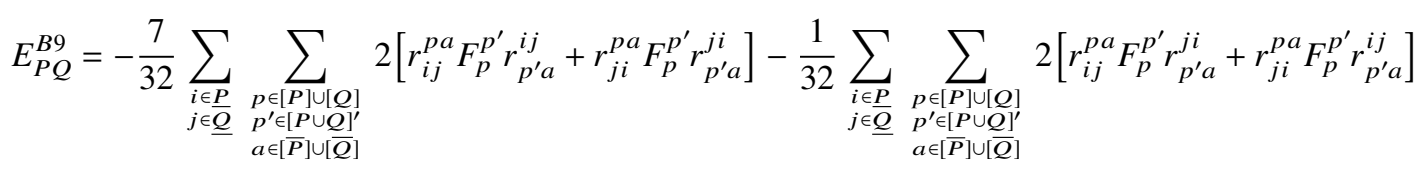

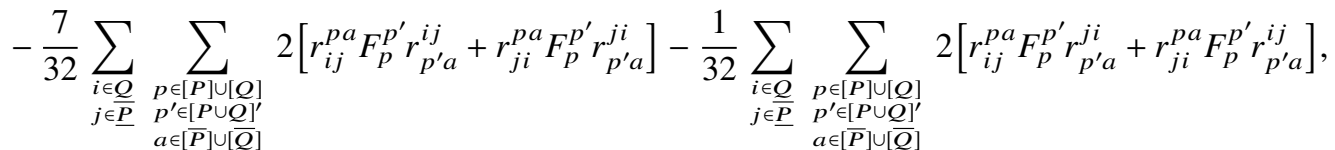

where $\{P\}^{\prime \prime} \equiv\{P\}_{\mathrm{CABSAO}+}$ and $\{P \cup Q\}^{\prime \prime} \equiv\{P\}^{\prime \prime} \cup\{Q\}^{\prime \prime}$. 
${ }^{1}$ I. Shavitt and R. J. Bartlett, Many-Body Methods in Chemistry and Physics (Cambridge University Press, 2009), Cambridge Books Online.

${ }^{2}$ T. Helgaker, P. Jørgensen, and J. Olsen, Molecular Electronic Structure Theory (Wiley, 2000).

${ }^{3}$ P. Pulay, Chem. Phys. Lett. 100, 151 (1983).

${ }^{4}$ S. Saebø and P. Pulay, Annu. Rev. Phys. Chem. 44, 213 (1993).

${ }^{5}$ C. Hampel and H.-J. Werner, J. Chem. Phys. 104, 6286 (1996).

${ }^{6}$ M. Schütz, G. Hetzer, and H.-J. Werner, J. Chem. Phys. 111, 5691 (1999).

${ }^{7}$ G. Hetzer, M. Schütz, H. Stoll, and H.-J. Werner, J. Chem. Phys. 113, 9443 (2000).

${ }^{8}$ C. Edmiston and M. Krauss, J. Chem. Phys. 42, 1119 (1965).

${ }^{9}$ W. Meyer, J. Chem. Phys. 58, 1017 (1973).

${ }^{10}$ F. Neese, F. Wennmohs, and A. Hansen, J. Chem. Phys. 130, 114108 (2009).

${ }^{11}$ F. Neese, A. Hansen, and D. G. Liakos, J. Chem. Phys. 131, 064103 (2009).

${ }^{12}$ J. Yang, Y. Kurashige, F. R. Manby, and G. K. L. Chan, J. Chem. Phys. 134, 044123 (2011).

${ }^{13}$ G. Schmitz, B. Helmich, and C. Hättig, Mol. Phys. 111, 2463 (2013).

${ }^{14}$ D. Usvyat, L. Maschio, and M. Schütz, J. Chem. Phys. 143, 102805 (2015).

${ }^{15}$ K. Kitaura, E. Ikeo, T. Asada, T. Nakano, and M. Uebayasi, Chem. Phys. Lett. 313, 701 (1999).

${ }^{16}$ D. G. Fedorov, K. Ishimura, T. Ishida, K. Kitaura, P. Pulay, and S. Nagase, J. Comput. Chem. 28, 1476 (2007).

${ }^{17}$ G. D. Fletcher, D. G. Fedorov, S. R. Pruitt, T. L. Windus, and M. S. Gordon, J. Chem. Theory Comput. 8, 75 (2012).

${ }^{18}$ R. K. Nesbet, Phys. Rev. 155, 51 (1967)

${ }^{19}$ H. Stoll, Ann. Phys. 508, 355 (1996).

${ }^{20}$ J. Friedrich, M. Hanrath, and M. Dolg, J. Chem. Phys. 126, 154110 (2007).

${ }^{21} \mathrm{~S}$. Li, J. Ma, and Y. Jiang, J. Comput. Chem. 23, 237 (2002).

${ }^{22} \mathrm{~W}$. Li and S. Li, Sci. Chin. Chem. 57, 78 (2013).

${ }^{23}$ M. Kállay, J. Chem. Phys. 142, 204105 (2015).

${ }^{24}$ M. Ziolkowski, B. Jansik, T. Kjærgaard, and P. Jørgensen, J. Chem. Phys. 133, 014107 (2010).

${ }^{25}$ K. Kristensen, M. Ziolkowski, B. Jansik, T. Kjærgaard, and P. Jørgensen, J. Chem. Theory Comput. 7, 1677 (2011).

${ }^{26}$ LSDalton (local version), a linear scaling molecular electronic structure program, Release Dalton2016, see http://daltonprogram.org. (2015).

${ }^{27}$ K. Kristensen, P. Jørgensen, B. Jansik, T. Kjærgaard, and S. Reine, J. Chem. Phys. 137, 114102 (2012).

${ }^{28}$ K. Kristensen, I.-M. Høyvik, B. Jansik, P. Jørgensen, T. Kjærgaard, S. Reine, and J. Jakowski, Phys. Chem. Chem. Phys. 14, 15706 (2012).

${ }^{29}$ I.-M. Høyvik, K. Kristensen, B. Jansik, and P. Jørgensen, J. Chem. Phys. 136, 014105 (2012).

${ }^{30}$ K. Kristensen, T. Kjærgaard, I.-M. Høyvik, P. Ettenhuber, P. Jørgensen, B. Jansik, S. Reine, and J. Jakowski, Mol. Phys. 111, 1196 (2013).

${ }^{31}$ J. J. Eriksen, P. Baudin, P. Ettenhuber, K. Kristensen, T. Kjærgaard, and P. Jørgensen, J. Chem. Theory Comput. 11, 2984 (2015).

${ }^{32} \mathrm{P}$. Ettenhuber, P. Baudin, T. Kjærgaard, P. Jørgensen, and K. Kristensen, J. Chem. Phys. 144, 164116 (2016).

${ }^{33}$ H.-J. Werner, T. B. Adler, and F. R. Manby, J. Chem. Phys. 126, 164102 (2007).

${ }^{34}$ W. Kutzelnigg and J. D. Morgan, J. Chem. Phys. 96, 4484 (1992).

${ }^{35}$ D. S. Hollman, J. J. Wilke, and H. F. Schaefer, J. Chem. Phys. 138, 064107 (2013).

${ }^{36}$ T. Kato, Commun. Pure Appl. Math. 10, 151 (1957).

${ }^{37}$ E. A. Hylleraas, Z. Phys. 54, 347 (1929).

${ }^{38}$ W. Kutzelnigg, Theor. Chim. Acta 68, 445 (1985).

${ }^{39}$ W. Kutzelnigg and W. Klopper, J. Chem. Phys. 94, 1985 (1991).

${ }^{40}$ V. Termath, W. Klopper, and W. Kutzelnigg, J. Chem. Phys. 94, 2002 (1991).

${ }^{41}$ D. G. Liakos, R. Izsák, E. F. Valeev, and F. Neese, Mol. Phys. 111, 2653 (2013).

${ }^{42}$ K. Patkowski, J. Chem. Phys. 137, 034103 (2012).

${ }^{43}$ C. Hättig, W. Klopper, A. Köhn, and D. P. Tew, Chem. Rev. 112, 4 (2012).
${ }^{44}$ L. Kong, F. A. Bischoff, and E. F. Valeev, Chem. Rev. 112, 75 (2012).

${ }^{45}$ S. Ten-no and J. Noga, Wiley Interdiscip. Rev.: Comput. Mol. Sci. 2, 114 (2012).

${ }^{46}$ S. Ten-no, J. Chem. Phys. 121, 117 (2004).

${ }^{47}$ R. A. Bachorz, F. A. Bischoff, A. Glöß, C. Hättig, S. Höfener, W. Klopper, and D. P. Tew, J. Comput. Chem. 32, 2492 (2011).

${ }^{48}$ W. Klopper and C. C. M. Samson, J. Chem. Phys. 116, 6397 (2002).

${ }^{49}$ E. F. Valeev, Chem. Phys. Lett. 395, 190 (2004).

${ }^{50}$ A. R. Kendall and A. H. Früchtl, Theor. Chem. Acc. 97, 158 (1997).

${ }^{51}$ F. R. Manby, J. Chem. Phys. 119, 4607 (2003).

${ }^{52}$ G. Schmitz, C. Hättig, and D. P. Tew, Phys. Chem. Chem. Phys. 16, 22167 (2014).

${ }^{53}$ J. Friedrich, D. P. Tew, W. Klopper, and M. Dolg, J. Chem. Phys. 132, 164114 (2010).

${ }^{54}$ J. Friedrich and J. Hänchen, J. Chem. Theory Comput. 9, 5381 (2013).

${ }^{55}$ F. Pavošević, F. Neese, and E. F. Valeev, J. Chem. Phys. 141, 054106 (2014).

${ }^{56}$ H.-J. Werner and F. R. Manby, J. Chem. Phys. 124, 054114 (2006).

${ }^{57}$ T. B. Adler, H.-J. Werner, and F. R. Manby, J. Chem. Phys. 130, 054106 (2009).

${ }^{58}$ D. P. Tew, B. Helmich, and C. Hättig, J. Chem. Phys. 135, 074107 (2011).

${ }^{59}$ H.-J. Werner, J. Chem. Phys. 129, 101103 (2008).

${ }^{60}$ T. B. Adler and H.-J. Werner, J. Chem. Phys. 130, 241101 (2009).

${ }^{61}$ H.-J. Werner, G. Knizia, C. Krause, M. Schwilk, and M. Dornbach, J. Chem. Theory Comput. 11, 484 (2015).

${ }^{62}$ Q. Ma and H.-J. Werner, J. Chem. Theory Comput. 11, 5291 (2015).

${ }^{63}$ P. Baudin, P. Ettenhuber, S. Reine, K. Kristensen, and T. Kjærgaard, J. Chem. Phys. 144, 054102 (2016).

${ }^{64}$ S. Kedžuch, M. Milko, and J. Noga, Int. J. Quantum Chem. 105, 929 (2005).

${ }^{65}$ S. Ten-no, Chem. Phys. Lett. 398, 56 (2004).

${ }^{66}$ B. J. Persson and P. R. Taylor, J. Chem. Phys. 105, 5915 (1996).

${ }^{67}$ D. P. Tew and W. Klopper, J. Chem. Phys. 123, 074101 (2005).

${ }^{68}$ K. A. Peterson, T. B. Adler, and H.-J. Werner, J. Chem. Phys. 128, 084102 (2008).

${ }^{69}$ B. Jansik, S. Høst, K. Kristensen, and P. Jørgensen, J. Chem. Phys. 134, 194104 (2011)

${ }^{70}$ I.-M. Høyvik, B. Jansik, and P. Jørgensen, J. Chem. Theory Comput. 8, 3137 (2012).

${ }^{71}$ I.-M. Høyvik, B. Jansik, K. Kristensen, and P. Jørgensen, J. Comput. Chem. 34, 1311 (2013).

${ }^{72}$ B. I. Dunlap, Phys. Chem. Chem. Phys. 2, 2113 (2000).

${ }^{73}$ See supplementary material at http://dx.doi.org/10.1063/1.4951696 for geometries of test set molecules.

${ }^{74}$ J. G. Hill and K. A. Peterson, Phys. Chem. Chem. Phys. 12, 10460 (2010).

${ }^{75}$ F. Weigend, A. Köhn, and C. Hättig, J. Chem. Phys. 116, 3175 (2002).

${ }^{76}$ C. Hättig, Phys. Chem. Chem. Phys. 7, 59 (2005).

${ }^{77}$ K. Aidas, C. Angeli, K. L. Bak, V. Bakken, R. Bast, L. Boman, O. Christiansen, R. Cimiraglia, S. Coriani, P. Dahle, E. K. Dalskov, U. Ekström, T. Enevoldsen, J. J. Eriksen, P. Ettenhuber, B. Fernández, L. Ferrighi, H. Fliegl, L. Frediani, K. Hald, A. Halkier, C. Hättig, H. Heiberg, T. Helgaker, A. C. Hennum, H. Hettema, E. Hjertenæs, S. Høst, I.-M. Høyvik, M. F. Iozzi, B. Jansik, H. J. A. Jensen, D. Jonsson, P. Jørgensen, J. Kauczor, S. Kirpekar, T. Kjærgaard, W. Klopper, S. Knecht, R. Kobayashi, H. Koch, J. Kongsted, A. Krapp, K. Kristensen, A. Ligabue, O. B. Lutnæs, J. I. Melo, K. V. Mikkelsen, R. H. Myhre, C. Neiss, C. B. Nielsen, P. Norman, J. Olsen, J. M. H. Olsen, A. Osted, M. J. Packer, F. Pawlowski, T. B. Pedersen, P. F. Provasi, S. Reine, Z. Rinkevicius, T. A. Ruden, K. Ruud, V. V. Rybkin, P. Salek, C. C. M. Samson, A. S. de Merás, T. Saue, S. P. A. Sauer, B. Schimmelpfennig, K. Sneskov, A. H. Steindal, K. O. Sylvester-Hvid, P. R. Taylor, A. M. Teale, E. I. Tellgren, D. P. Tew, A. J. Thorvaldsen, L. Thøgersen, O. Vahtras, M. A. Watson, D. J. D. Wilson, M. Ziolkowski, and H. Àgren, Wiley Interdiscip. Rev.: Comput. Mol. Sci. 4, 269 (2014). 